\title{
The Social Demography of Covid-19 Delta
}

\section{Charles Crothers}

School of Social Sciences

AUT

October, 2021

\section{Introduction}

The trajectory of Covid and its immediate consequences are tracked across several official and some non-official data sources. The main data comes from $\mathrm{MOH}$, with the reported data related to:

- Scanning

- Testing

- Vaccination

- Cases of Covid and also

- MIQ.

Supplementary data provided by Stats NZ and MBIE and covers labour force and hardships consequences, besides other information. In addition, a range of surveys (some carried out by government agencies) provide information on people's attitudes (see Crothers, 2021 for a summary). The usefulness of these various sources of information depends on the speed with which they are processed and made available and the extent to which the data allows close examination of particular social groupings given the social breakdowns provided. Fortunately, $\mathrm{MOH}$ data in particular is quickly available and although social breakdowns have been limited more has been made available recently, although ways of presenting data differ according to source. In a fast-changing situation the data presented here will be quickly out of date although it is likely that some of the broad patterns will endure.

Given the wider range of data available and the small-area spatial scale, Vaccination data affords the best insight into social effects on Covid-related phenomena. At the DHB level having $3 \mathrm{DHBs}$ each with somewhat different social characteristics provides some insight into socio-economic effects.

\section{Literature}

There is already a small literature = which is useful since $\mathrm{MOH}$ present but do not analyses their data. A media report and two analyses reveal effects of systematic racism in terms of Covid outcomes - both hospitalisations and deaths (Janesen, 2021; Steyn et al., 2021a; Steyn 2021b) while a study of the spatial arrangements of vaccination services (Whitehead et al, 2021) comes to the same conclusion. 


\section{Scanning}

Data has been developed for various ways of scanning and monthly averages shown in the table that the long period thought which NZ did not experience cases tended to suppress New Zealanders' scanning behaviour.

\section{Report}

\begin{tabular}{|c|c|c|c|c|c|c|c|}
\hline \multicolumn{8}{|l|}{ Mean } \\
\hline Period & $\begin{array}{c}\text { App } \\
\text { Registrations } \\
\end{array}$ & $\begin{array}{l}\text { QR Codes } \\
\text { Generated }\end{array}$ & $\begin{array}{c}\text { NZBN } \\
\text { Registered } \\
\text { Businesses } \\
\end{array}$ & Scans & $\begin{array}{l}\text { Manual } \\
\text { Entries }\end{array}$ & $\begin{array}{c}\text { Active } \\
\text { Devices }\end{array}$ & $\begin{array}{c}\text { Bluetooth } \\
\text { Active (24hr) }\end{array}$ \\
\hline 2020-05 & 36615.3846 & $2,578.38$ & $18,981.50$ & 32241.6154 & 00 & & \\
\hline 2020-06 & 3666.6667 & $2,126.88$ & 733.84 & 29441.8667 & 00 & $10,293.13$ & \\
\hline 2020-07 & 1654.5455 & 294.64 & 101.77 & 18936.3871 & 317.7419 & $10,270.90$ & \\
\hline 2020-08 & 50014.2857 & $9,376.36$ & $3,316.89$ & 920085.5161 & 67661.1935 & $416,437.97$ & \\
\hline 2020-09 & 950000 & $1,536.50$ & 178.11 & 1677153.8333 & 4600000 & $735,849.63$ & \\
\hline $2020-10$ & 1506.4516 & 651.74 & 43.68 & 768900.5161 & 28281.7742 & $377,089.03$ & \\
\hline 2020-11 & 181000 & 783.67 & 59.70 & 873032.9333 & 30711.3333 & $418,983.23$ & \\
\hline 2020-12 & 144400 & 398.83 & 34.37 & 515588.3226 & 20372.6452 & $270,508.61$ & $411,821.77$ \\
\hline 2021-01 & 3925.4000 & 845.23 & 63.33 & 661939.8387 & 30712.1613 & $351,297.52$ & $630,327.73$ \\
\hline 2021-02 & 5846.2143 & $2,001.89$ & 160.64 & 1215640.3929 & 41744.5714 & $622,617.93$ & $1,074,020.30$ \\
\hline 2021-03 & 2026.9355 & $1,004.19$ & 71.19 & 1185040.9355 & 28915.4194 & $611,710.13$ & $1,247,662.50$ \\
\hline 2021-04 & 1156.6333 & 545.10 & 30.33 & 726707.8333 & 26165.1333 & 386,399 & $1,289,125.87$ \\
\hline 2021-05 & 1081.6129 & 472.74 & 24.48 & 584357.9677 & 23909.4516 & $314,948.03$ & $1,303,754.32$ \\
\hline 2021-06 & 1469.9000 & 547.67 & 37.43 & 602275.2667 & 34222.6000 & $322,850.13$ & $1,445,668.60$ \\
\hline 2021-07 & 848.6452 & 464.65 & 28.06 & 641844.8065 & 28786.2581 & $338,765.61$ & $1,492,569.19$ \\
\hline 2021-08 & 6981.8065 & $2,377.71$ & 257.61 & 603584.2258 & 85937.4516 & $391,959.06$ & $1,663,231.29$ \\
\hline 2021-09 & 4634.4000 & $2,136.43$ & 269.47 & 2139209.8000 & 76954.0333 & $1,037,450.90$ & $2,100,572.33$ \\
\hline $2021-10$ & 2314.1818 & $1,652.23$ & 117.82 & 2367558.8636 & 66139.7727 & $1,130,387.27$ & $2,199,154.68$ \\
\hline Total & 6617.1756 & $1,608.53$ & 471.75 & 863767.5230 & 36029.1820 & $462,017.96$ & $1,384,854.24$ \\
\hline
\end{tabular}

\section{Testing:}

$\mathrm{MOH}$ tables show higher testing rates for the 3 Auckland DHBs, and also higher proportions of tests which are positive. The peak age-range are 40 year olds and there is the slightest gender difference. 
All tests by DHB and in managed isolation and quarantine facilities from 22 January 2020 to 24 October 2021

\begin{tabular}{|c|c|c|c|}
\hline & Total tests & \begin{tabular}{|l} 
Tested \\
positive (\%)
\end{tabular} & $\begin{array}{l}\text { Test rate per } 1000 \\
\text { people }\end{array}$ \\
\hline Total & 3923269 & $0.18 \%$ & 697.75 \\
\hline \multicolumn{4}{|l|}{ Location } \\
\hline Managed isolation and quarantine & 381208 & $0.45 \%$ & NA \\
\hline Auckland & 581241 & $0.17 \%$ & 1037.01 \\
\hline Bay of Plenty & 128797 & $0.07 \%$ & 526.66 \\
\hline Canterbury & 271685 & $0.07 \%$ & 466.31 \\
\hline Capital and Coast & 180517 & $0.09 \%$ & 554.91 \\
\hline Counties Manukau & 740081 & $0.22 \%$ & 1276.34 \\
\hline Hawkes Bay & 64266 & $0.08 \%$ & 382.79 \\
\hline Hutt Valley & 60959 & $0.05 \%$ & 401.49 \\
\hline Lakes & 59799 & $0.04 \%$ & 535.53 \\
\hline MidCentral & 74585 & $0.05 \%$ & 408.44 \\
\hline Nelson Marlborough & 63368 & $0.08 \%$ & 414.2 \\
\hline Northland & 109300 & $0.06 \%$ & 594.17 \\
\hline South Canterbury & 19098 & $0.18 \%$ & 317.47 \\
\hline Southern & 136953 & $0.24 \%$ & 406.23 \\
\hline Tairāwhiti & 21046 & $0.02 \%$ & 425.79 \\
\hline Taranaki & 49407 & $0.03 \%$ & 405.99 \\
\hline Waikato & 262034 & $0.12 \%$ & 610.69 \\
\hline Wairarapa & 16572 & $0.06 \%$ & 359.3 \\
\hline Waitematā & 643106 & $0.18 \%$ & 997.15 \\
\hline West Coast & 5681 & $0.07 \%$ & 177.86 \\
\hline Whanganui & 20721 & $0.03 \%$ & 318.51 \\
\hline Unknown & 32845 & $0.25 \%$ & NA \\
\hline \multicolumn{4}{|l|}{ Ethnicity ${ }^{1}$} \\
\hline Māori & 529768 & $0.22 \%$ & 669.46 \\
\hline Pacific peoples & 474704 & $0.31 \%$ & 1458.86 \\
\hline Asian & 666468 & $0.16 \%$ & 830.74 \\
\hline European/Other & 2098885 & $0.13 \%$ & 674.81 \\
\hline Unknown & 153444 & $0.35 \%$ & NA \\
\hline \multicolumn{4}{|l|}{ Age group } \\
\hline 0 to 9 & 265037 & $0.28 \%$ & 420 \\
\hline 10 to 19 & 346490 & $0.23 \%$ & 540.56 \\
\hline 20 to 29 & 765901 & $0.2 \%$ & 1025.43 \\
\hline 30 to 39 & 748434 & $0.18 \%$ & 1097.45 \\
\hline 40 to 49 & 591918 & $0.16 \%$ & 972.19 \\
\hline 50 to 59 & 580953 & $0.15 \%$ & 915.61 \\
\hline 60 to 69 & 392753 & $0.12 \%$ & 734.36 \\
\hline 70 to 79 & 160085 & $0.11 \%$ & 440.35 \\
\hline $80+$ & 71626 & $0.1 \%$ & 383.68 \\
\hline
\end{tabular}

\footnotetext{
${ }^{1}$ The prioritised ethnicity classification system is used which means each person is allocated to a single ethnic group, based on the ethnic groups they identify with. Where people identify with more than one group, they are assigned in this order of priority: Māori, Pacific Peoples, Asian, and European/Other. So, if a person identifies as being Māori and New Zealand European, the person is counted as Māori.
} 
All tests by DHB and in managed isolation and quarantine facilities from 22 January 2020 to 24 October 2021

\begin{tabular}{|l|l|l|l|}
\hline & Total tests & $\begin{array}{l}\text { Tested } \\
\text { positive (\%) }\end{array}$ & $\begin{array}{l}\text { Test rate per 1000 } \\
\text { people }\end{array}$ \\
\hline Sex & & & \\
\hline Female & 1990622 & $0.16 \%$ & 782.39 \\
\hline Male & 1894648 & $0.19 \%$ & 762.42 \\
\hline
\end{tabular}

\section{Vaccination:}

$\mathrm{MOH}$ has been releasing vaccination date data by $\operatorname{DHB}(n=20)$ and locality (SA2; $n=c 2139)$. Data are up to $17^{\text {th }}$ Oct 2021. The DHB level data is not only age (and gender specific) but also includes both Level 1 and Level 2 ethnic levels, allowing quite detailed tracking of groups. The table below is highly summarised but shows that for the $65+$ age-group rates are similar for the three ethnic groupings, with increasing divergence with successively younger age-groups.

\begin{tabular}{|l|l|l|l|l|l|l|} 
Age-group & $65+$ & $50-64$ & $35-49$ & $20-34$ & $12-19$ & $\begin{array}{l}\text { All Ages } \\
(12+)\end{array}$ \\
\hline Maori D1 & 94 & 82 & 67 & 54 & 57 & 66 \\
\hline Maori 2 & 85 & 68 & 45 & 28 & 30 & 45 \\
Pacific p 1 & 95 & 95 & 89 & 84 & 74 & 74 \\
Pacific p 2 & 89 & 89 & 77 & 65 & 47 & 45 \\
Other 1 & 95 & 90 & 87 & 85 & 84 & 88 \\
Other 2 & 89 & 78 & 66 & 56 & 53 & 70 \\
\hline
\end{tabular}

The locality-level data allow many links to be made, but they are links at the 'aggregate level' and such links might not also hold at the individual level. At the locality level separate data is provided for all, Maori and Pacific rates: from these an 'Other (neither Maori nor Pacific) rate can be calculated. This is much higher than that for either Pacifica or Maori, which are both alarmingly low. Given that $2^{\text {nd }}$ dose tends to follow fairly 'automatically' from the first attention is focused on the $1^{\text {st }}$. 
SA2 Descriptive Statistics rates per Million

\begin{tabular}{l|r|r|r|r|r} 
& N & \multicolumn{1}{c}{ Minimum } & Maximum & \multicolumn{1}{c}{ Mean } & \multicolumn{1}{c}{ Std. Deviation } \\
\hline Dose rate 1 & 2112 & 200 & 950 & 781.1430 & 95.80597 \\
\hline Dose rate 2 & 2143 & 161 & 949 & 491.1213 & 94.55336 \\
& & & & & \\
& & & & & \\
\hline Maori dose rate 1 & 2120 & 167 & 949 & 625.7943 & 123.51036 \\
\hline Maori dose rate 2 & 2127 & 83 & 762 & 361.0282 & 102.23506 \\
\hline Pacific dose rate 1 & 1853 & 91 & 950 & 703.7593 & 134.21954 \\
\hline Pacific dose rat e2 & 1968 & 53 & 944 & 440.3943 & 138.34085 \\
\hline Other dose rate 1 & 1596 & 79 & 6079 & 1497.6610 & 740.86173 \\
\hline Other dose rate 2 & 1596 & 56 & 3937 & 951.3490 & 478.58694 \\
\hline
\end{tabular}

Regional groupings have very similar overall rates with the South of the North Island lagging overall and for other but not so much for Maori (not-significant) or Pacifica. Measures of Association are not high apart slightly for others.

\begin{tabular}{|c|c|c|c|c|c|}
\hline \multicolumn{6}{|c|}{ Report } \\
\hline \multicolumn{2}{|c|}{ Region group } & $\begin{array}{c}\text { Overall dose r } \\
1 \\
\end{array}$ & Maori dose r1 & $\begin{array}{c}\text { Pacifica dose } \\
\text { r1 } \\
\end{array}$ & other dose r1 \\
\hline \multirow[t]{3}{*}{$\mathrm{NNI}$} & Mean & 784.0825 & 629.2097 & 714.8568 & 855.4225 \\
\hline & $\mathrm{N}$ & 1006 & 1011 & 936 & 846 \\
\hline & Std. Deviation & 103.52029 & 125.76191 & 124.39512 & 77.70373 \\
\hline \multirow[t]{3}{*}{ SNI } & Mean & 770.9498 & 620.3923 & 697.6879 & 832.9471 \\
\hline & $\mathrm{N}$ & 518 & 520 & 455 & 383 \\
\hline & Std. Deviation & 97.32019 & 123.09177 & 138.02683 & 69.24244 \\
\hline \multirow[t]{3}{*}{ SI } & Mean & 784.8279 & 624.3231 & 687.2554 & 820.4314 \\
\hline & $\mathrm{N}$ & 587 & 588 & 462 & 366 \\
\hline & Std. Deviation & 78.52870 & 119.69495 & 147.19369 & 69.48057 \\
\hline \multirow[t]{3}{*}{ Total } & Mean & 781.1430 & 625.7943 & 703.7593 & 841.9963 \\
\hline & $\mathrm{N}$ & 2112 & 2120 & 1853 & 1595 \\
\hline & Std. Deviation & 95.80597 & 123.51036 & 134.21954 & 75.34792 \\
\hline
\end{tabular}

\begin{tabular}{lllr} 
& & & \multicolumn{2}{c}{ Sig. } \\
\hline Overall dose rate 1 * Region & Between Groups & (Combined) & .015 \\
\cline { 3 - 4 } & & Linearity & .142 \\
\cline { 2 - 4 } & & Deviation from Linearity & .016 \\
\cline { 2 - 4 } & Within Groups & & \\
\cline { 2 - 4 } & Total & &
\end{tabular}




\begin{tabular}{|c|c|c|c|}
\hline \multirow{5}{*}{$\begin{array}{l}\text { Maori dose rate } 1{ }^{*} \text { Region } \\
\text { group }\end{array}$} & \multirow[t]{3}{*}{ Between Groups } & (Combined) & .168 \\
\hline & & Linearity & .185 \\
\hline & & Deviation from Linearity & .192 \\
\hline & \multicolumn{3}{|l|}{ Within Groups } \\
\hline & \multicolumn{3}{|l|}{ Total } \\
\hline \multirow{5}{*}{$\begin{array}{l}\text { Pacifica dose rate } 1 \text { * Region } \\
\text { group }\end{array}$} & \multirow[t]{3}{*}{ Between Groups } & (Combined) & 1 \\
\hline & & Linearity & 0 \\
\hline & & Deviation from Linearity & .646 \\
\hline & \multicolumn{3}{|l|}{ Within Groups } \\
\hline & \multicolumn{3}{|l|}{ Total } \\
\hline \multirow{5}{*}{$\begin{array}{l}\text { Other dose rate } 1{ }^{*} \text { Region } \\
\text { group }\end{array}$} & \multirow[t]{3}{*}{ Between Groups } & (Combined) & 0 \\
\hline & & Linearity & 0 \\
\hline & & Deviation from Linearity & .261 \\
\hline & \multicolumn{3}{|l|}{ Within Groups } \\
\hline & \multicolumn{3}{|l|}{ Total } \\
\hline
\end{tabular}

\section{Measures of Association}

\begin{tabular}{l|r|r|r|r} 
& $\mathrm{R}$ & $\mathrm{R}$ Squared & Eta & Eta Squared \\
\hline $\begin{array}{l}\text { Overall dose rate 1 * Region } \\
\text { group }\end{array}$ & .032 & 1 & .070 & 5 \\
\hline $\begin{array}{l}\text { Maori dose rate 1 * Region } \\
\text { group }\end{array}$ & .029 & 1 & .049 & 2 \\
\hline $\begin{array}{l}\text { Pacifica dose rate 1 } \\
\text { group } \text { Region }\end{array}$ & -.087 & 8 & .088 & 8 \\
\hline $\begin{array}{l}\text { Other dose rate 1 * Region } \\
\text { group }\end{array}$ & -.196 & .038 & .198 & .039 \\
\hline
\end{tabular}

There is a clear fall-off with size of urban area, illustrated by quite strong measures of association.

\begin{tabular}{|c|c|c|c|c|c|}
\hline \multicolumn{6}{|c|}{ Report } \\
\hline Urban Influence & & Overall dose r1 & Maori dose r1 & $\begin{array}{c}\text { Pacifica dose } \\
\text { r1 }\end{array}$ & Other dose $\mathrm{r} 1$ \\
\hline \multirow[t]{3}{*}{ Major urban area } & Mean & 833.1362 & 671.7698 & 743.0205 & 882.3315 \\
\hline & $\mathrm{N}$ & 859 & 873 & 830 & 815 \\
\hline & Std. Deviation & 77.94581 & 126.71987 & 99.59948 & 60.97583 \\
\hline \multirow[t]{3}{*}{ Large urban area } & Mean & 755.0927 & 587.3874 & 701.7778 & 813.6019 \\
\hline & $\mathrm{N}$ & 302 & 302 & 279 & 264 \\
\hline & Std. Deviation & 93.61640 & 111.07191 & 119.99332 & 60.75898 \\
\hline Medium urban area & Mean & 780.2414 & 607.2315 & 705.3315 & 809.7080 \\
\hline
\end{tabular}




\begin{tabular}{|c|c|c|c|c|c|}
\hline & $\mathrm{N}$ & 203 & 203 & 178 & 154 \\
\hline & Std. Deviation & 74.73634 & 99.18734 & 113.69678 & 59.26543 \\
\hline \multirow[t]{3}{*}{ High urban accessibility } & Mean & 806.1392 & 689.3974 & 715.3333 & 834.5475 \\
\hline & $\mathrm{N}$ & 79 & 78 & 66 & 58 \\
\hline & Std. Deviation & 66.76360 & 114.87230 & 161.45753 & 46.72130 \\
\hline \multirow[t]{3}{*}{ Medium-High } & Mean & 785.0732 & 668.3902 & 634.3103 & 802.5513 \\
\hline & $\mathrm{N}$ & 41 & 41 & 29 & 21 \\
\hline & Std. Deviation & 52.64332 & 98.61260 & 187.12927 & 41.82065 \\
\hline \multirow[t]{3}{*}{ Medium urban accessibility } & Mean & 752.4088 & 610.4173 & 662.7087 & 799.2125 \\
\hline & $\mathrm{N}$ & 137 & 139 & 103 & 86 \\
\hline & Std. Deviation & 77.03662 & 105.70145 & 158.15775 & 57.03383 \\
\hline \multirow[t]{3}{*}{ Lo-Medium } & Mean & 727.4786 & 582.5517 & 634.2347 & 767.4006 \\
\hline & $\mathrm{N}$ & 117 & 116 & 98 & 43 \\
\hline & Std. Deviation & 68.17556 & 96.23082 & 157.41905 & 51.85334 \\
\hline \multirow[t]{3}{*}{ Low urban accessibility } & Mean & 718.8480 & 565.6512 & 673.3953 & 770.4387 \\
\hline & $\mathrm{N}$ & 171 & 172 & 129 & 90 \\
\hline & Std. Deviation & 84.68662 & 103.37704 & 154.63553 & 70.03889 \\
\hline \multirow[t]{3}{*}{ Remote } & Mean & 705.5068 & 564.1857 & 580.0816 & 784.8055 \\
\hline & $\mathrm{N}$ & 73 & 70 & 49 & 28 \\
\hline & Std. Deviation & 111.75806 & 113.16197 & 168.98778 & 58.51038 \\
\hline \multirow[t]{3}{*}{ Remote/V remote } & Mean & 686.5484 & 530.5161 & 552.8182 & 722.7282 \\
\hline & $\mathrm{N}$ & 31 & 31 & 22 & 7 \\
\hline & Std. Deviation & 108.10576 & 104.40749 & 209.73195 & 128.96466 \\
\hline \multirow[t]{3}{*}{ Very remote } & Mean & 701.8235 & 55800 & 671.8000 & 763.0940 \\
\hline & $\mathrm{N}$ & 17 & 17 & 15 & 9 \\
\hline & Std. Deviation & 90.34049 & 75.18311 & 168.89693 & 50.67599 \\
\hline \multirow[t]{3}{*}{ Total } & Mean & 781.1430 & 625.7943 & 703.7593 & 841.9963 \\
\hline & $\mathrm{N}$ & 2112 & 2120 & 1853 & 1595 \\
\hline & Std. Deviation & 95.80597 & 123.51036 & 134.21954 & 75.34792 \\
\hline
\end{tabular}

\begin{tabular}{lllr} 
& & & Sig. \\
\hline Overall dose rate 1 & Between Groups & (Combined) & 0 \\
\cline { 3 - 4 } & & Linearity & 0 \\
\cline { 2 - 4 } & & & 0 \\
\cline { 2 - 4 } & Weviation from Linearity & 0 \\
\cline { 2 - 4 } & Within Groups & & 0 \\
\hline Maori dose rate 1 & Between Groups & (Combined) & 0 \\
UrbInfl & & Linearity & 0
\end{tabular}




\begin{tabular}{|c|c|c|c|}
\hline & Within Groups & & \\
\hline & Total & & \\
\hline \multirow{5}{*}{$\begin{array}{l}\text { Pacifica dose rate } 1 \text { * } \\
\text { UrbInfl }\end{array}$} & Between Groups & (Combined) & 0 \\
\hline & & Linearity & 0 \\
\hline & & Deviation from Linearity & 0 \\
\hline & Within Groups & & \\
\hline & Total & & \\
\hline \multirow{5}{*}{$\begin{array}{l}\text { Other dose rate } 1^{*} \\
\text { UrbInfl }\end{array}$} & Between Groups & (Combined) & 0 \\
\hline & & Linearity & 0 \\
\hline & & Deviation from Linearity & 0 \\
\hline & Within Groups & & \\
\hline & Total & & \\
\hline
\end{tabular}

Measures of Association

\begin{tabular}{lr|r|r|r} 
& R & R Squared & \multicolumn{1}{|c|}{ Eta } & Eta Squared \\
\hline $\begin{array}{l}\text { Overall dose rate 1 * } \\
\text { UrbInfl }\end{array}$ & -.390 & .152 & .529 & .280 \\
\hline $\begin{array}{l}\text { Maori dose rate 1 * } \\
\text { UrbInfl }\end{array}$ & -.205 & .042 & .383 & .147 \\
\hline $\begin{array}{l}\text { Pacifica dose rate 1 } \\
\text { UrbInfl }\end{array}$ & -.287 & .082 & .357 & .128 \\
\hline $\begin{array}{l}\text { Other dose rate 1 * } \\
\text { UrbInfl }\end{array}$ & -.414 & .171 & .600 & .360 \\
\hline
\end{tabular}

There is an even stronger association (although less so for Pacifica) between Deprivation level of area and vaccination.

\section{Report}

\begin{tabular}{|c|c|c|c|c|c|}
\hline \multicolumn{2}{|c|}{ Dep Index 2018} & $\begin{array}{c}\text { Overall dose } \\
\text { rate } 1\end{array}$ & $\begin{array}{c}\text { Maori dose rate } \\
1 \\
\end{array}$ & $\begin{array}{c}\text { Pacifica dose } \\
\text { rate } 1\end{array}$ & $\begin{array}{c}\text { Other dose rate } \\
1 \\
\end{array}$ \\
\hline \multirow[t]{3}{*}{1} & Mean & 862.8244 & 757.0194 & 74400 & 885.5966 \\
\hline & $\mathrm{N}$ & 205 & 206 & 161 & 139 \\
\hline & Std. Deviation & 47.85505 & 103.81310 & 145.30167 & 42.19815 \\
\hline \multirow[t]{3}{*}{2} & Mean & 838.2123 & 713.6143 & 732.7584 & 864.1221 \\
\hline & $\mathrm{N}$ & 212 & 210 & 178 & 155 \\
\hline & Std. Deviation & 56.73032 & 104.13641 & 141.88474 & 50.88380 \\
\hline \multirow[t]{3}{*}{3} & Mean & 833.0141 & 695.8302 & 721.5503 & 866.4080 \\
\hline & $\mathrm{N}$ & 213 & 212 & 189 & 159 \\
\hline & Std. Deviation & 63.97674 & 109.99116 & 142.59813 & 50.76826 \\
\hline
\end{tabular}




\begin{tabular}{|c|c|c|c|c|c|}
\hline \multirow[t]{3}{*}{4} & Mean & 816.1564 & 655.2394 & 717.9259 & 855.2799 \\
\hline & $\mathrm{N}$ & 211 & 213 & 189 & 155 \\
\hline & Std. Deviation & 69.32476 & 101.41147 & 131.53397 & 58.51600 \\
\hline \multirow[t]{3}{*}{5} & Mean & 799.2536 & 632.7867 & 710.7486 & 845.8585 \\
\hline & $\mathrm{N}$ & 209 & 211 & 179 & 159 \\
\hline & Std. Deviation & 76.69456 & 97.93383 & 144.10015 & 74.28837 \\
\hline \multirow[t]{3}{*}{6} & Mean & 776.8942 & 608.5261 & 706.6339 & 836.6411 \\
\hline & $\mathrm{N}$ & 208 & 211 & 183 & 147 \\
\hline & Std. Deviation & 75.07078 & 99.28954 & 124.65576 & 69.64919 \\
\hline \multirow[t]{3}{*}{7} & Mean & 766.7000 & 584.0427 & 709.1064 & 823.8223 \\
\hline & $\mathrm{N}$ & 210 & 211 & 188 & 168 \\
\hline & Std. Deviation & 73.49524 & 93.67641 & 108.77249 & 70.62662 \\
\hline \multirow[t]{3}{*}{8} & Mean & 758.0191 & 573.5896 & 709.2995 & 827.7679 \\
\hline & $\mathrm{N}$ & 209 & 212 & 187 & 174 \\
\hline & Std. Deviation & 83.12444 & 90.48844 & 109.19966 & 80.29763 \\
\hline \multirow[t]{3}{*}{9} & Mean & 717.7512 & 546.2958 & 661.5052 & 820.2692 \\
\hline & $\mathrm{N}$ & 205 & 213 & 194 & 167 \\
\hline & Std. Deviation & 75.16962 & 85.65366 & 134.12917 & 100.75419 \\
\hline \multirow[t]{3}{*}{10} & Mean & 653.0892 & 498.6462 & 641.5545 & 806.5319 \\
\hline & $\mathrm{N}$ & 213 & 212 & 202 & 172 \\
\hline & Std. Deviation & 94.40937 & 75.54982 & 123.61322 & 90.55114 \\
\hline \multirow[t]{3}{*}{ Total } & Mean & 782.1189 & 626.1071 & 704.1573 & 841.9963 \\
\hline & $\mathrm{N}$ & 2095 & 2111 & 1850 & 1595 \\
\hline & Std. Deviation & 93.87658 & 122.77768 & 133.94250 & 75.34792 \\
\hline
\end{tabular}

Sig.

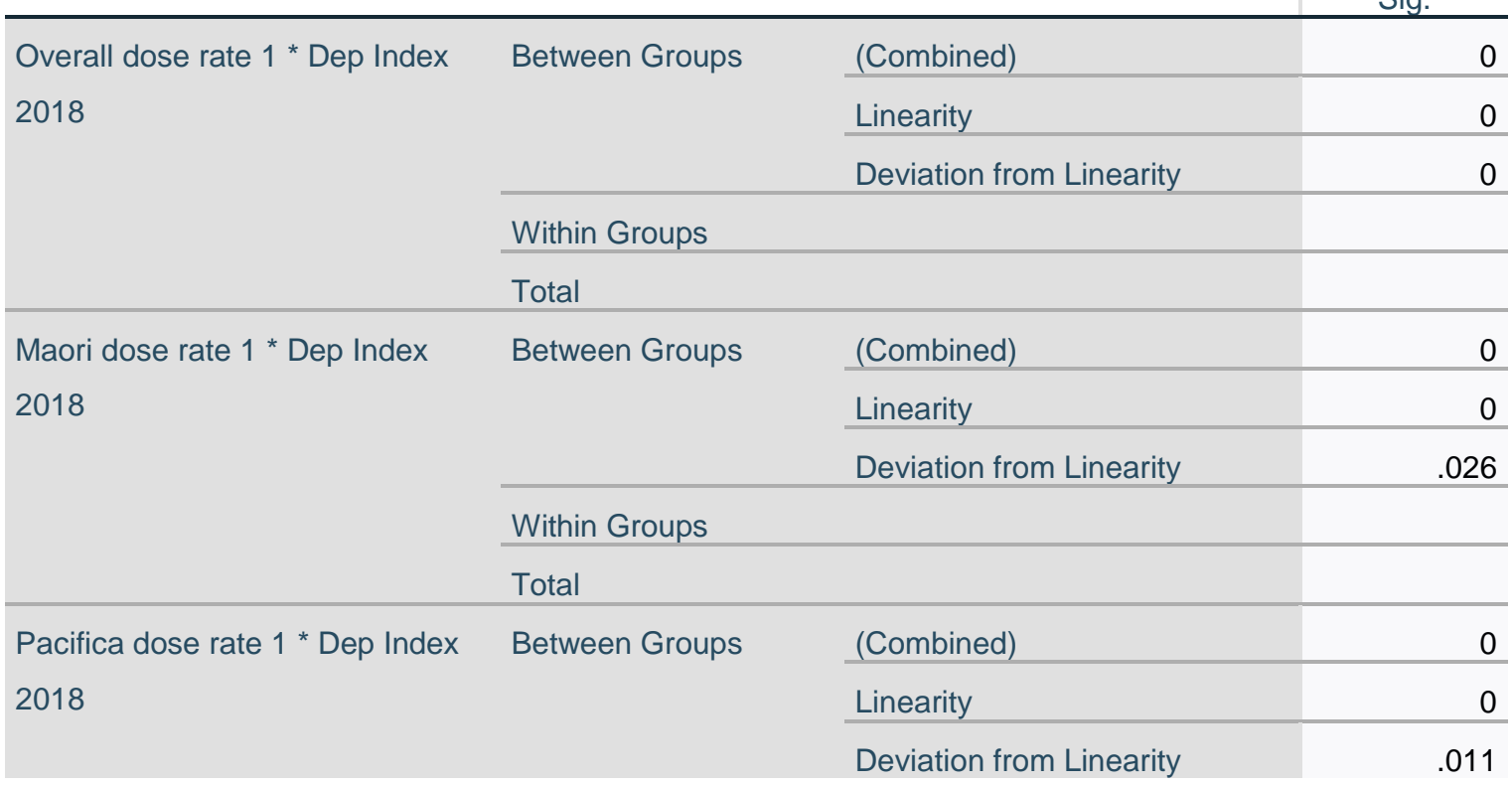




\begin{tabular}{|c|c|c|c|}
\hline & Within Groups & & \\
\hline & Total & & \\
\hline \multirow{5}{*}{$\begin{array}{l}\text { Other dose rate } 1 \text { * Dep Index } \\
2018\end{array}$} & Between Groups & (Combined) & 0 \\
\hline & & Linearity & 0 \\
\hline & & Deviation from Linearity & .596 \\
\hline & Within Groups & & \\
\hline & Total & & \\
\hline
\end{tabular}

Measures of Association

\begin{tabular}{lr|r|r|r} 
& $\mathrm{R}$ & \multicolumn{1}{|c|}{ R Squared } & \multicolumn{1}{c|}{ Eta } & Eta Squared \\
\hline $\begin{array}{l}\text { Overall dose rate 1 * Dep Index } \\
2018\end{array}$ & -.609 & .371 & .635 & .403 \\
\hline $\begin{array}{l}\text { Maori dose rate 1 * Dep Index } \\
2018\end{array}$ & -.615 & .378 & .619 & .383 \\
\hline $\begin{array}{l}\text { Pacifica dose rate 1 * Dep Index } \\
2018\end{array}$ & -.199 & .040 & .224 & .050 \\
\hline $\begin{array}{l}\text { Other dose rate 1 * Dep Index } \\
2018\end{array}$ & -.301 & .091 & .307 & .094 \\
\hline
\end{tabular}

Some illustrative graphs are followed by a table of correlations of by rates with appropriate locality characteristics.

The four vaccination rates are very strongly locked together which suggests that localitylevel characteristics may be pushing all in the locality towards a similar rate. Regions yielded little difference (see also above) in terms of tenure (except for other), local authority ownership, those without vehicles especially for Maori but not Pacifica, population change rate, median age, \% European, \% New Zealander, length of residence, average household size

Relations were strong with Density (moderately and not so much for Polynesian) and especially dep index, together with other measures of socio-economic status (median rent, affluence scale, private landlord, Housing NZ ownership (esp. overall or Maori) iwi ownership, access to the internet, living in joined dwelling, \% Asian, MELAA, being born in NZ, variably able to spe4ak English, Personal Income. Smoking regularly, \% Professionals, agriculture workers, working at home (strongly negative) couple with children households, single parent households (negative) , single person household, relativized median income, \% crowded. 

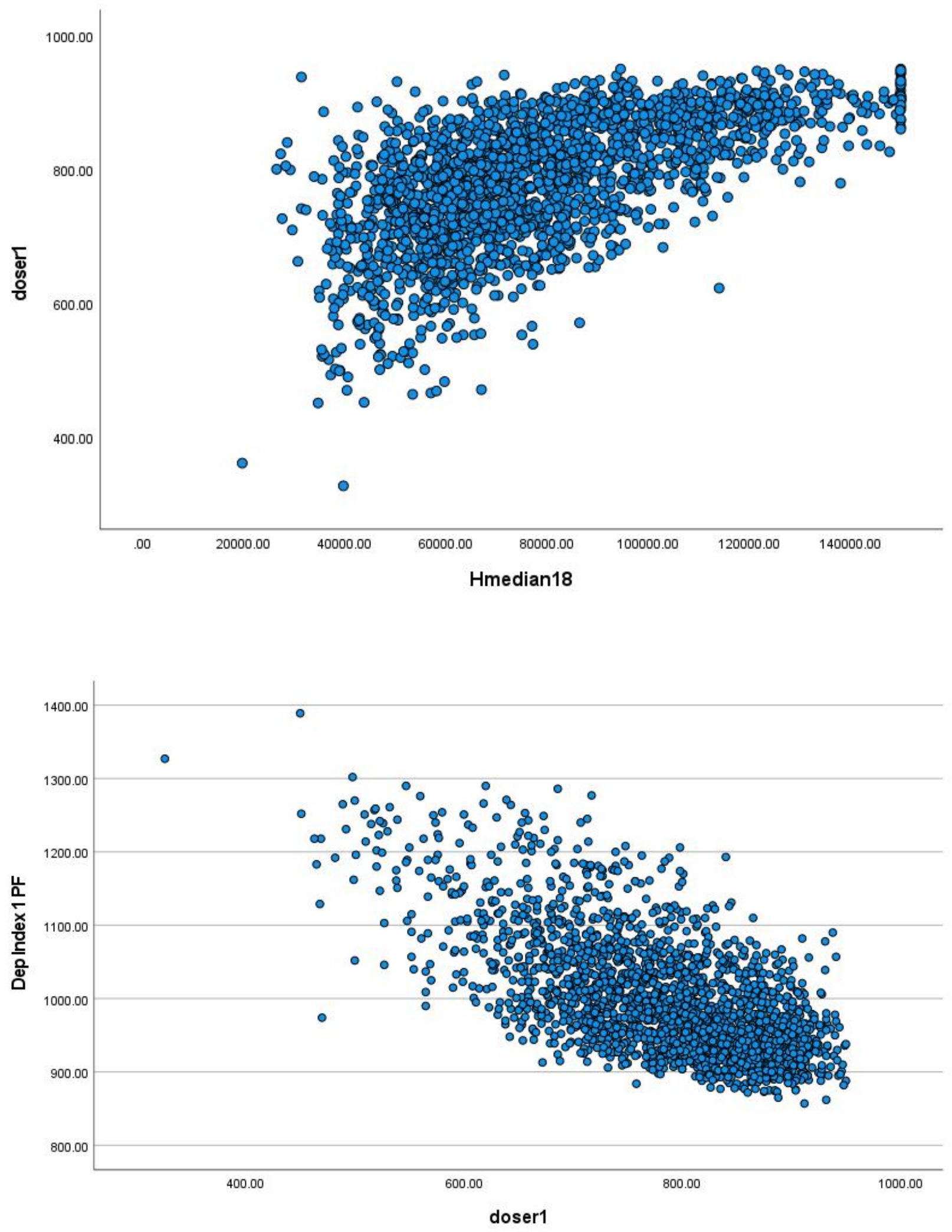


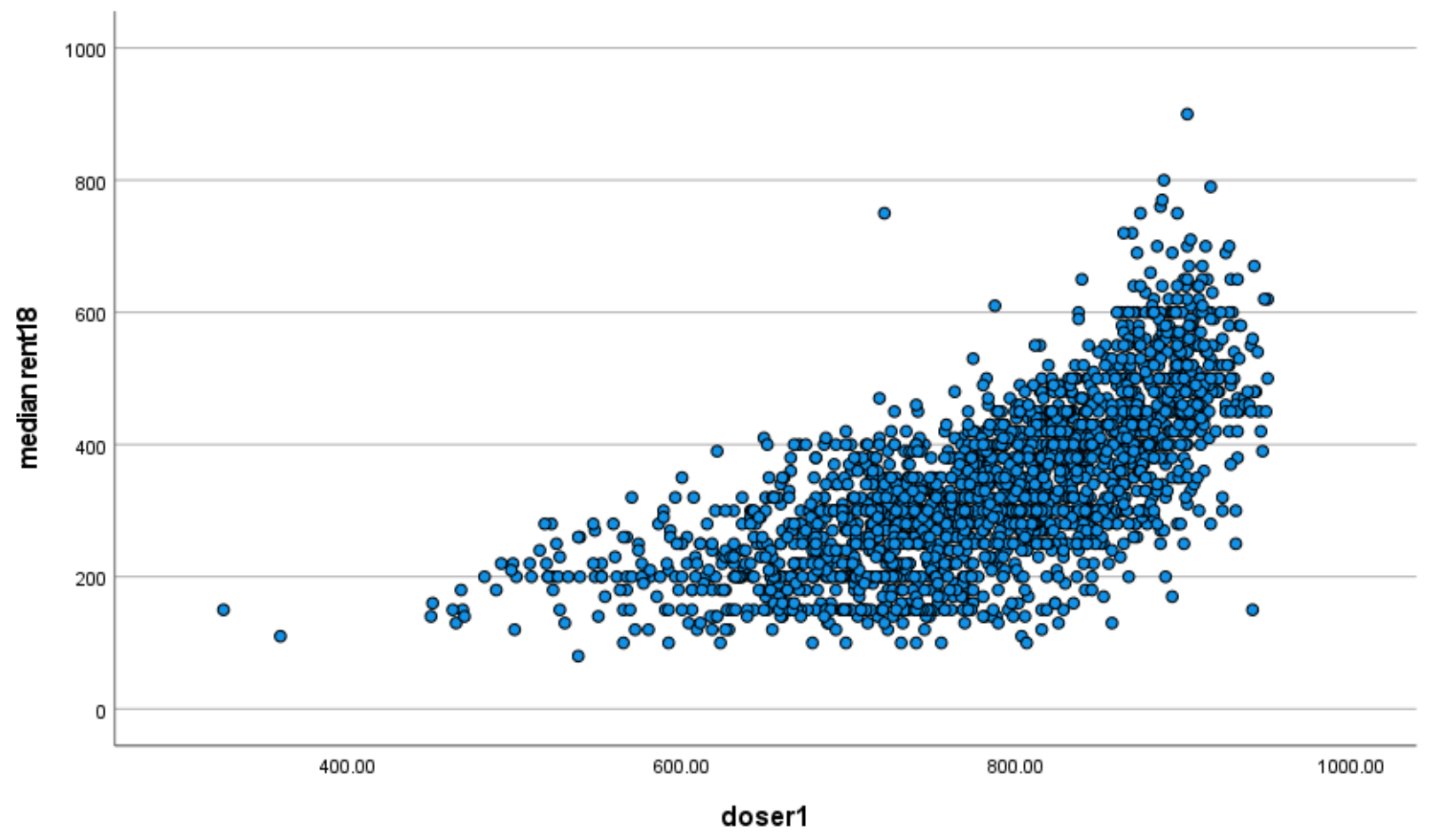

Graph

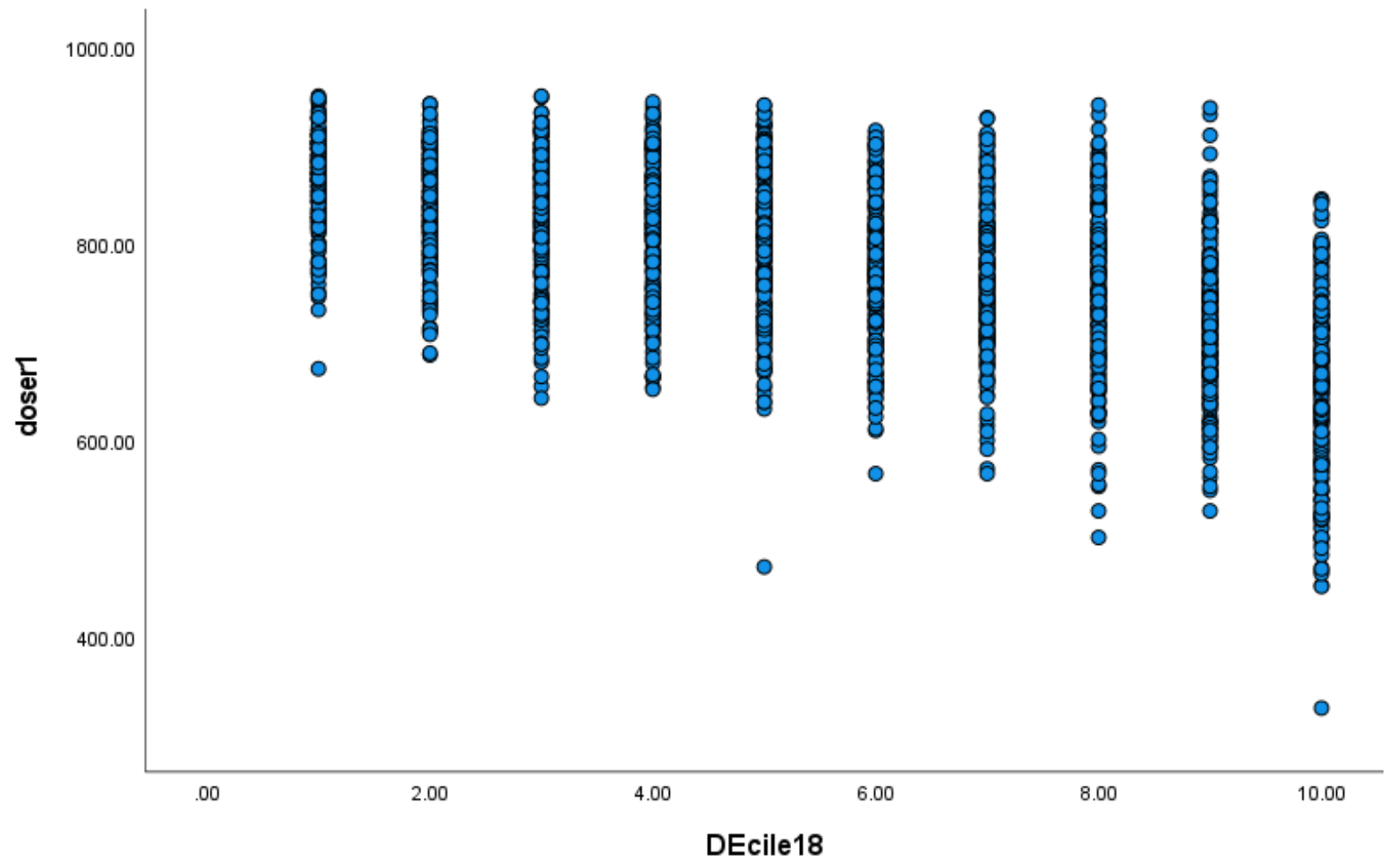




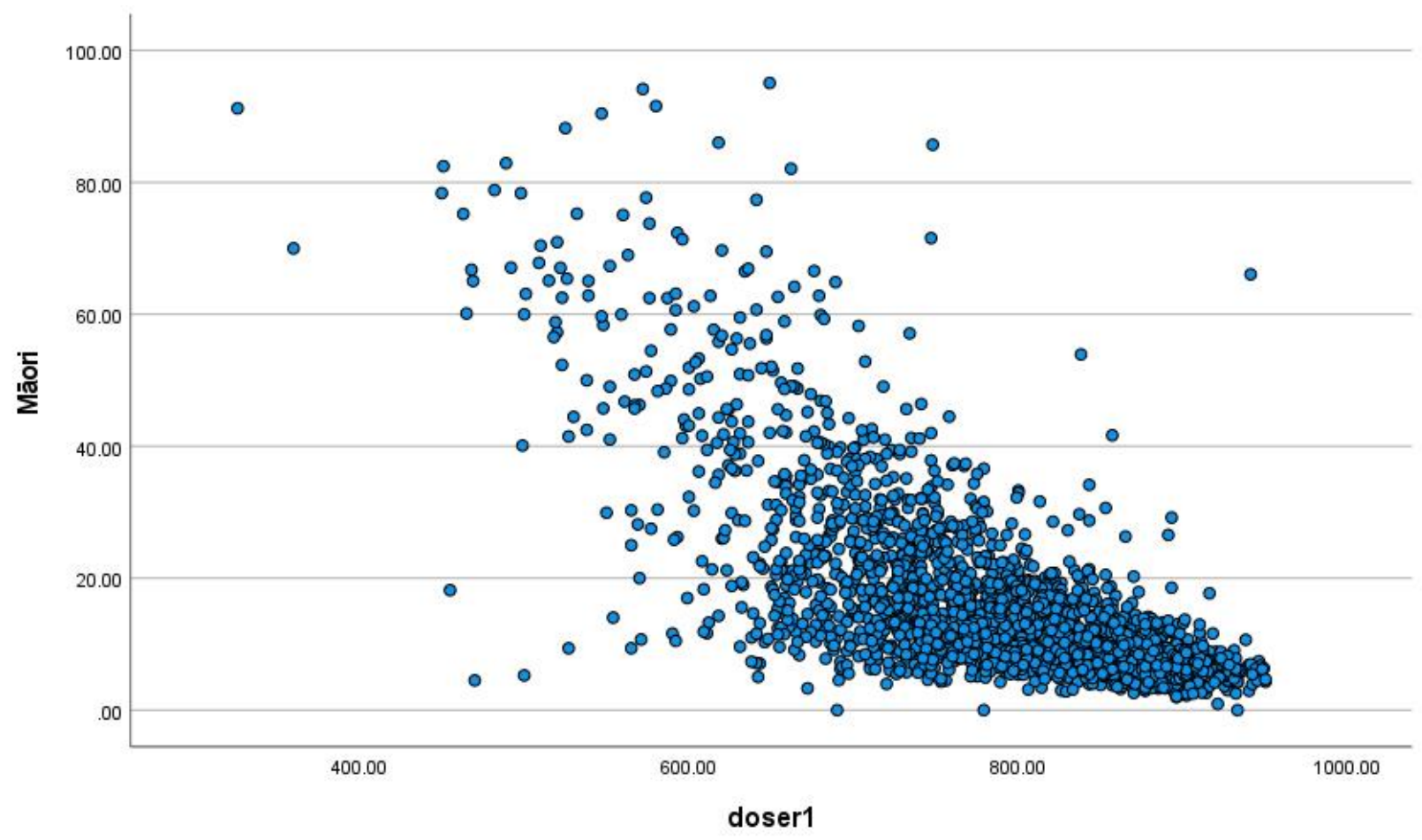

\begin{tabular}{|c|c|c|c|c|c|}
\hline & & $\begin{array}{c}\text { Overall dose } \\
\text { r1 }\end{array}$ & $\begin{array}{c}\text { Maori dose } \\
\text { r1 } \\
\end{array}$ & $\begin{array}{l}\text { Pacifica } \\
\text { dose r1 }\end{array}$ & $\begin{array}{c}\text { Other } \\
\text { dose } r 1 \\
\end{array}$ \\
\hline \multirow[t]{3}{*}{ Overall dose rate 1} & Pearson Correlation & 1 & $.775^{* *}$ & $.458^{* *}$ & $.838^{* *}$ \\
\hline & Sig. (2-tailed) & & 0 & 0 & 0 \\
\hline & $\mathrm{N}$ & 2112 & 2089 & 1832 & 1566 \\
\hline \multirow[t]{3}{*}{ Maori dose rate 1} & Pearson Correlation & $.775^{\star *}$ & 1 & $.371^{* *}$ & $.576^{* *}$ \\
\hline & Sig. (2-tailed) & 0 & & 0 & 0 \\
\hline & $\mathrm{N}$ & 2089 & 2120 & 1843 & 1585 \\
\hline \multirow[t]{3}{*}{ Pacifica dose rate 1} & Pearson Correlation & $.458^{* *}$ & $.371^{* *}$ & 1 & $.348^{* *}$ \\
\hline & Sig. (2-tailed) & 0 & 0 & & 0 \\
\hline & $\mathrm{N}$ & 1832 & 1843 & 1853 & 1458 \\
\hline \multirow[t]{3}{*}{ Other dose rate 1} & Pearson Correlation & $.838^{* *}$ & $.576^{* *}$ & $.348^{* *}$ & 1 \\
\hline & Sig. (2-tailed) & 0 & 0 & 0 & \\
\hline & $\mathrm{N}$ & 1566 & 1585 & 1458 & 1595 \\
\hline \multirow[t]{3}{*}{ Region group } & Pearson Correlation & .032 & .029 & $-.087^{* *}$ & $-.196^{\star *}$ \\
\hline & Sig. (2-tailed) & .142 & .185 & 0 & 0 \\
\hline & $\mathrm{N}$ & 2112 & 2120 & 1853 & 1595 \\
\hline \multirow[t]{3}{*}{ Density } & Pearson Correlation & $.332^{\star \star}$ & $.124^{\star *}$ & $.179^{\star *}$ & $.445^{\star \star}$ \\
\hline & Sig. (2-tailed) & 0 & 0 & 0 & 0 \\
\hline & $\mathrm{N}$ & 2108 & 2119 & 1853 & 1595 \\
\hline \multirow[t]{2}{*}{ Dep Index 2018} & Pearson Correlation & $-.609^{* \star}$ & $-.615^{* *}$ & $-.199^{* *}$ & $-.301^{* *}$ \\
\hline & Sig. (2-tailed) & 0 & 0 & 0 & 0 \\
\hline
\end{tabular}




\begin{tabular}{|c|c|c|c|c|c|}
\hline & $\mathrm{N}$ & 2095 & 2111 & 1850 & 1595 \\
\hline \multirow[t]{3}{*}{ median rent18 } & Pearson Correlation & $.679^{* *}$ & $.584^{* *}$ & $.374^{* *}$ & $.576 *$ \\
\hline & Sig. (2-tailed) & 0 & 0 & 0 & 0 \\
\hline & $\mathrm{N}$ & 2095 & 2112 & 1851 & 1595 \\
\hline \multirow[t]{3}{*}{ Affluence scale } & Pearson Correlation & $.645^{* *}$ & $.666^{* *}$ & $.213^{* *}$ & $.427^{* *}$ \\
\hline & Sig. (2-tailed) & 0 & 0 & 0 & 0 \\
\hline & $\mathrm{N}$ & 2101 & 2114 & 1853 & 1595 \\
\hline \multirow{3}{*}{$\begin{array}{l}\text { Total Dwelling owned or partly } \\
\text { owned } 2018\end{array}$} & Pearson Correlation & -.033 & $.095^{* *}$ & -.026 & $-.354^{* *}$ \\
\hline & Sig. (2-tailed) & .128 & 0 & .269 & 0 \\
\hline & $\mathrm{N}$ & 2098 & 2114 & 1851 & 1595 \\
\hline \multirow{3}{*}{$\begin{array}{l}\text { Private person trust or business } \\
2018\end{array}$} & Pearson Correlation & $.282^{* *}$ & $.314^{* *}$ & $.047^{*}$ & $.064^{*}$ \\
\hline & Sig. (2-tailed) & 0 & 0 & .041 & .011 \\
\hline & $\mathrm{N}$ & 2095 & 2112 & 1851 & 1595 \\
\hline \multirow[t]{3}{*}{ Local authority or city council } & Pearson Correlation & $-.072^{* *}$ & $-.086^{* *}$ & -.017 & $-.142^{* *}$ \\
\hline & Sig. (2-tailed) & 1 & 0 & .469 & 0 \\
\hline & $\mathrm{N}$ & 2069 & 2089 & 1844 & 1593 \\
\hline \multirow[t]{3}{*}{ Housing New Zealand Corporation } & Pearson Correlation & $-.209^{* *}$ & $-.272^{* *}$ & -.020 & -2 \\
\hline & Sig. (2-tailed) & 0 & 0 & .401 & .937 \\
\hline & $\mathrm{N}$ & 2069 & 2089 & 1844 & 1593 \\
\hline \multirow[t]{3}{*}{ Iwi hapū or Māori land trust } & Pearson Correlation & $-.250^{* *}$ & $-.132^{* *}$ & $-.175^{* *}$ & $-.158^{* *}$ \\
\hline & Sig. (2-tailed) & 0 & 0 & 0 & 0 \\
\hline & $\mathrm{N}$ & 2069 & 2089 & 1844 & 1593 \\
\hline \multirow[t]{3}{*}{ Other community housing provider } & Pearson Correlation & $-.082^{* *}$ & $-.116^{* *}$ & -2 & $-.082^{* *}$ \\
\hline & Sig. (2-tailed) & 0 & 0 & .935 & 1 \\
\hline & $\mathrm{N}$ & 2069 & 2089 & 1844 & 1593 \\
\hline \multirow{3}{*}{$\begin{array}{l}\text { Other state owned corporation or } \\
\text { state owned enterprise or } \\
\text { government department or ministry }\end{array}$} & Pearson Correlation & $-.113^{* *}$ & $-.069^{* *}$ & -.030 & -5 \\
\hline & Sig. (2-tailed) & 0 & 2 & .197 & .848 \\
\hline & $\mathrm{N}$ & 2069 & 2089 & 1844 & 1593 \\
\hline \multirow[t]{3}{*}{ No motor vehicle 2018} & Pearson Correlation & $-.064^{* *}$ & $-.123^{* *}$ & .027 & $.191^{* *}$ \\
\hline & Sig. (2-tailed) & 3 & 0 & .252 & 0 \\
\hline & $\mathrm{N}$ & 2094 & 2111 & 1849 & 1595 \\
\hline \multirow[t]{3}{*}{ Access to the internet } & Pearson Correlation & $.702^{* *}$ & $.597^{* *}$ & $.311^{* *}$ & $.505^{* *}$ \\
\hline & Sig. (2-tailed) & 0 & 0 & 0 & 0 \\
\hline & $\mathrm{N}$ & 2099 & 2115 & 1852 & 1595 \\
\hline \multirow[t]{3}{*}{ Joined dwelling } & Pearson Correlation & $.300^{* *}$ & $.116^{* *}$ & $.147^{* *}$ & $.383^{* *}$ \\
\hline & Sig. (2-tailed) & 0 & 0 & 0 & 0 \\
\hline & $\mathrm{N}$ & 2108 & 2118 & 1852 & 1595 \\
\hline \multirow[t]{3}{*}{ Pop Chg 2008-18 \% } & Pearson Correlation & $.080^{* *}$ & $.070^{* *}$ & .037 & $.056^{*}$ \\
\hline & Sig. (2-tailed) & 0 & 1 & .113 & .025 \\
\hline & $\mathrm{N}$ & 2104 & 2114 & 1850 & 1590 \\
\hline
\end{tabular}




\begin{tabular}{|c|c|c|c|c|c|}
\hline \multirow[t]{3}{*}{ Median age (2018) } & Pearson Correlation & $.061^{* *}$ & $.088^{* *}$ & $-.069^{* *}$ & $-.160^{* *}$ \\
\hline & Sig. (2-tailed) & 5 & 0 & 3 & 0 \\
\hline & $\mathrm{N}$ & 2108 & 2119 & 1853 & 1595 \\
\hline \multirow[t]{3}{*}{ European } & Pearson Correlation & $.057^{* *}$ & $.141^{* *}$ & $-.085^{* *}$ & $-.358^{* *}$ \\
\hline & Sig. (2-tailed) & 9 & 0 & 0 & 0 \\
\hline & $\mathrm{N}$ & 2108 & 2119 & 1853 & 1595 \\
\hline \multirow[t]{3}{*}{ Māori } & Pearson Correlation & $-.714^{* *}$ & $-.499^{* *}$ & $-.258^{* *}$ & $-.499^{* *}$ \\
\hline & Sig. (2-tailed) & 0 & 0 & 0 & 0 \\
\hline & $\mathrm{N}$ & 2105 & 2118 & 1853 & 1595 \\
\hline \multirow[t]{3}{*}{ Pacific Peoples } & Pearson Correlation & $-.116^{* *}$ & $-.169^{* *}$ & .027 & $.179^{* *}$ \\
\hline & Sig. (2-tailed) & 0 & 0 & .246 & 0 \\
\hline & $\mathrm{N}$ & 2105 & 2118 & 1853 & 1595 \\
\hline \multirow[t]{3}{*}{ Asian } & Pearson Correlation & $.481^{\star *}$ & $.242^{* *}$ & $.270^{* *}$ & $.614^{\star *}$ \\
\hline & Sig. (2-tailed) & 0 & 0 & 0 & 0 \\
\hline & $\mathrm{N}$ & 2105 & 2118 & 1853 & 1595 \\
\hline \multirow[t]{3}{*}{ Middle Eastern Latin American } & Pearson Correlation & $.434^{\star *}$ & $.219^{* *}$ & $.213^{\star *}$ & $.514^{\star *}$ \\
\hline & Sig. (2-tailed) & 0 & 0 & 0 & 0 \\
\hline & $\mathrm{N}$ & 2105 & 2118 & 1853 & 1595 \\
\hline \multirow[t]{3}{*}{ Other Ethnicity } & Pearson Correlation & $.043^{*}$ & $.044^{*}$ & -.015 & $-.092^{\star *}$ \\
\hline & Sig. (2-tailed) & .047 & .044 & .507 & 0 \\
\hline & $\mathrm{N}$ & 2105 & 2118 & 1853 & 1595 \\
\hline \multirow[t]{3}{*}{ New Zealander } & Pearson Correlation & $-.066^{* *}$ & -4 & $-.094^{* *}$ & $-.243^{* \star}$ \\
\hline & Sig. (2-tailed) & 2 & .836 & 0 & 0 \\
\hline & $\mathrm{N}$ & 2103 & 2116 & 1853 & 1595 \\
\hline \multirow[t]{3}{*}{ New Zealand COB } & Pearson Correlation & $-.603^{* *}$ & $-.352^{* *}$ & $-.304^{* *}$ & $-.710^{* *}$ \\
\hline & Sig. (2-tailed) & 0 & 0 & 0 & 0 \\
\hline & $\mathrm{N}$ & 2108 & 2119 & 1853 & 1595 \\
\hline \multirow[t]{3}{*}{ \% Speak English } & Pearson Correlation & $-.218^{* *}$ & $-.052^{*}$ & $-.190^{\star *}$ & $-.421^{\star *}$ \\
\hline & Sig. (2-tailed) & 0 & .018 & 0 & 0 \\
\hline & $\mathrm{N}$ & 2108 & 2119 & 1853 & 1595 \\
\hline \multirow[t]{3}{*}{ \% Usual Residence 5 years } & Pearson Correlation & .023 & $.145^{* *}$ & -.026 & $-.169^{\star *}$ \\
\hline & Sig. (2-tailed) & .295 & 0 & .268 & 0 \\
\hline & $\mathrm{N}$ & 2106 & 2118 & 1853 & 1595 \\
\hline \multirow[t]{3}{*}{ Median \$ Personal income } & Pearson Correlation & $.535^{\star \star}$ & $.555^{* *}$ & $.223^{* \star}$ & $.337^{\star *}$ \\
\hline & Sig. (2-tailed) & 0 & 0 & 0 & 0 \\
\hline & $\mathrm{N}$ & 2112 & 2120 & 1853 & 1595 \\
\hline \multirow[t]{3}{*}{ No religion } & Pearson Correlation & -8 & $.123^{* *}$ & -.035 & $-.285^{* *}$ \\
\hline & Sig. (2-tailed) & .705 & 0 & .135 & 0 \\
\hline & $\mathrm{N}$ & 2108 & 2119 & 1853 & 1595 \\
\hline Christian & Pearson Correlation & .021 & -.033 & 2 & $.107^{\star *}$ \\
\hline
\end{tabular}




\begin{tabular}{|c|c|c|c|c|c|}
\hline & Sig. (2-tailed) & .335 & .124 & .920 & 0 \\
\hline & $\mathrm{N}$ & 2105 & 2118 & 1853 & 1595 \\
\hline \multirow[t]{3}{*}{ Regular smoker } & Pearson Correlation & $-.786^{* *}$ & $-.670^{* *}$ & $-.291^{* *}$ & $-.534^{* *}$ \\
\hline & Sig. (2-tailed) & 0 & 0 & 0 & 0 \\
\hline & $\mathrm{N}$ & 2108 & 2119 & 1853 & 1595 \\
\hline \multirow[t]{3}{*}{ Own/Trust } & Pearson Correlation & $.089^{* *}$ & $.194^{* *}$ & $-.057^{*}$ & $-.293^{* *}$ \\
\hline & Sig. (2-tailed) & 0 & 0 & .014 & 0 \\
\hline & $\mathrm{N}$ & 2108 & 2119 & 1853 & 1595 \\
\hline \multirow[t]{3}{*}{ Professionals } & Pearson Correlation & $.674^{* *}$ & $.642^{* *}$ & $.322^{* *}$ & $.531^{* *}$ \\
\hline & Sig. (2-tailed) & 0 & 0 & 0 & 0 \\
\hline & $\mathrm{N}$ & 2112 & 2120 & 1853 & 1595 \\
\hline \multirow{3}{*}{$\begin{array}{l}\text { Agriculture Forestry and Fishing } 18 \\
\text { WPLC }\end{array}$} & Pearson Correlation & $-.374^{* *}$ & $-.230^{* *}$ & $-.301^{* *}$ & $-.400^{* *}$ \\
\hline & Sig. (2-tailed) & 0 & 0 & 0 & 0 \\
\hline & $\mathrm{N}$ & 2112 & 2120 & 1853 & 1595 \\
\hline \multirow[t]{3}{*}{ Proportions (worked at home) } & Pearson Correlation & $-.274^{* *}$ & $-.137^{\star *}$ & $-.295^{* *}$ & $-.332 *$ \\
\hline & Sig. (2-tailed) & 0 & 0 & 0 & 0 \\
\hline & $\mathrm{N}$ & 2096 & 2111 & 1850 & 1595 \\
\hline \multirow{3}{*}{$\begin{array}{l}\text { Couple with children household } \\
\text { (with or without other people) }\end{array}$} & Pearson Correlation & $.289^{* *}$ & $.326^{* *}$ & $.142^{* *}$ & $.244^{* *}$ \\
\hline & Sig. (2-tailed) & 0 & 0 & 0 & 0 \\
\hline & $\mathrm{N}$ & 2073 & 2091 & 1844 & 1589 \\
\hline \multirow{3}{*}{$\begin{array}{l}\text { Single parent household (with or } \\
\text { without other people) }\end{array}$} & Pearson Correlation & $-.433^{* *}$ & $-.409^{* *}$ & $-.053^{*}$ & $-.270^{* *}$ \\
\hline & Sig. (2-tailed) & 0 & 0 & .023 & 0 \\
\hline & $\mathrm{N}$ & 2073 & 2091 & 1844 & 1589 \\
\hline \multirow[t]{3}{*}{ Other multi-person household } & Pearson Correlation & $.146^{* *}$ & $.078^{* *}$ & $.125^{* *}$ & $.285^{* *}$ \\
\hline & Sig. (2-tailed) & 0 & 0 & 0 & 0 \\
\hline & $\mathrm{N}$ & 2096 & 2112 & 1850 & 1595 \\
\hline \multirow[t]{3}{*}{ One-person household } & Pearson Correlation & $-.208^{* *}$ & $-.263^{* *}$ & $-.118^{* *}$ & $-.286^{* *}$ \\
\hline & Sig. (2-tailed) & 0 & 0 & 0 & 0 \\
\hline & $\mathrm{N}$ & 2096 & 2112 & 1850 & 1595 \\
\hline \multirow[t]{3}{*}{ Median_OECD_Modified_Income } & Pearson Correlation & $.631^{* *}$ & $.635^{* *}$ & $.263^{* *}$ & $.441^{* *}$ \\
\hline & Sig. (2-tailed) & 0 & 0 & 0 & 0 \\
\hline & $\mathrm{N}$ & 2103 & 2117 & 1852 & 1595 \\
\hline \multirow[t]{3}{*}{$40-50$ housing rental costs } & Pearson Correlation & $.045^{*}$ & 5 & .043 & $-.104^{* *}$ \\
\hline & Sig. (2-tailed) & .050 & .819 & .075 & 0 \\
\hline & $\mathrm{N}$ & 1910 & 1929 & 1743 & 1556 \\
\hline \multirow[t]{3}{*}{ Percent crowded } & Pearson Correlation & $-.197^{* *}$ & $-.234^{* *}$ & -7 & $.213^{* *}$ \\
\hline & Sig. (2-tailed) & 0 & 0 & .780 & 0 \\
\hline & $\mathrm{N}$ & 1952 & 1972 & 1758 & 1532 \\
\hline \multirow[t]{2}{*}{ Residents Ave } & Pearson Correlation & $.089^{* *}$ & $.101^{* *}$ & $.125^{* *}$ & $.296 *$ \\
\hline & Sig. (2-tailed) & 0 & 0 & 0 & 0 \\
\hline
\end{tabular}




\section{Catching Covid:}

Covid cases need to be sequestered between those in MIQ (almost totally drawn from overseas travellers) and 'community cases. During periods where there were no community cases were necessarily drawn from overseas. Community cases are drawn substantially from each of the main ethnic groups and across age groups up to the 50s and even between the two genders. Those hospitalized have ben older and with more males.

\section{period * miq Status Crosstabulation}

$\%$ within period

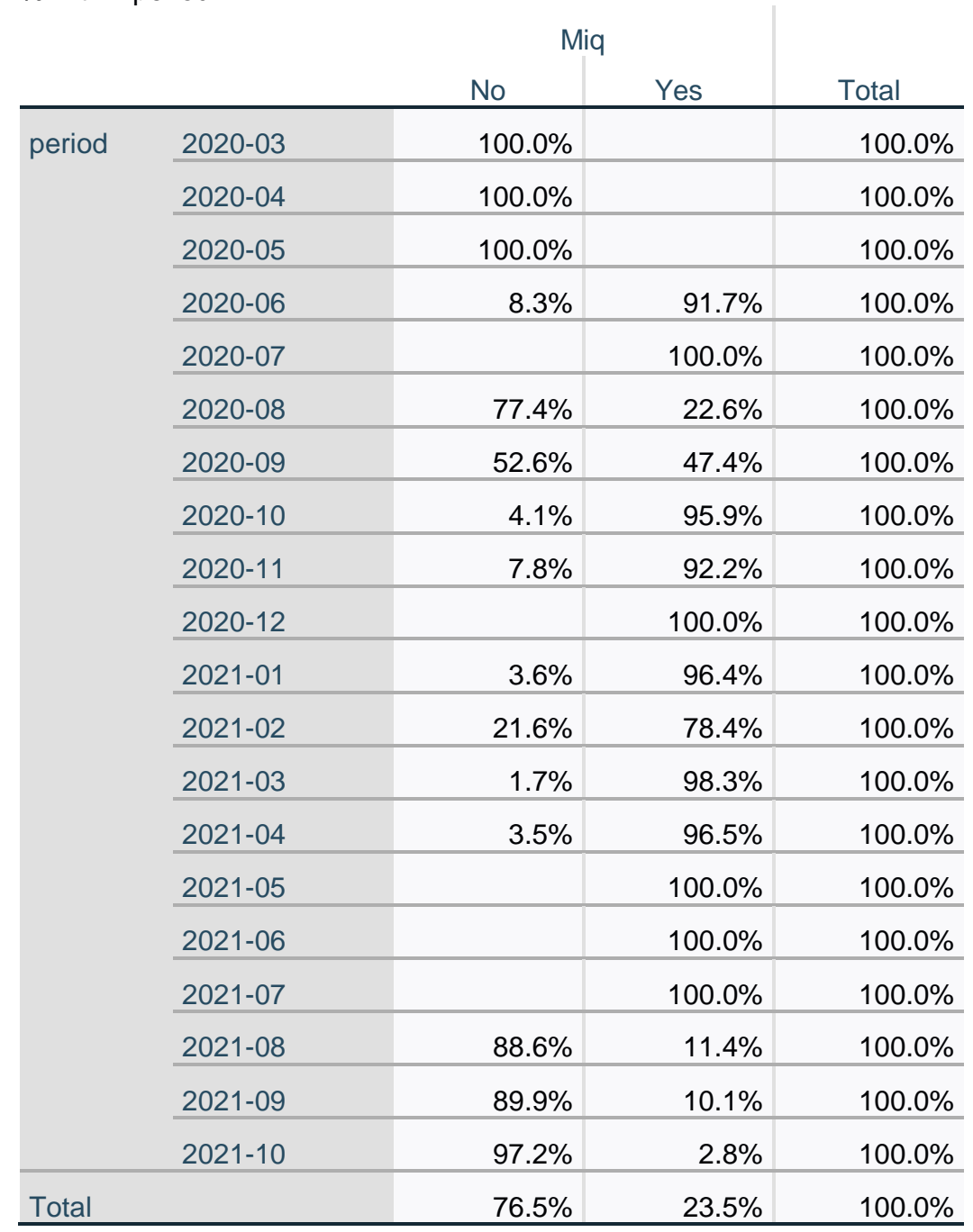

Nearly one-third of overseas travellers do not get assigned to MIQ. Very few community cases are assigned to MIQ. 


\section{Overseas travel * miq Crosstabulation}

$\%$ within Overseas travel

\begin{tabular}{llr|r|r} 
& \multicolumn{3}{c|}{ miq } & \\
\hline \multirow{2}{*}{ Overseas travel } & No & \multicolumn{1}{c}{ yes } & \multicolumn{1}{c}{ Total } \\
\cline { 2 - 5 } & No & $99.5 \%$ & $0.5 \%$ & $100.0 \%$ \\
\cline { 2 - 5 } & Unknown & $98.7 \%$ & $1.3 \%$ & $100.0 \%$ \\
\cline { 2 - 5 } & Yes & $30.7 \%$ & $69.3 \%$ & $100.0 \%$ \\
\hline \multirow{2}{*}{ Total } & & $76.5 \%$ & $23.5 \%$ & $100.0 \%$ \\
\hline
\end{tabular}

\begin{tabular}{|c|c|c|c|c|c|c|c|}
\hline & & Other & Waitemata & Auckland & $\begin{array}{l}\text { Counties- } \\
\text { Manukau }\end{array}$ & Waikato & \\
\hline \multirow[t]{15}{*}{ period } & 2020-03 & $50.1 \%$ & $13.6 \%$ & $13.6 \%$ & $8.3 \%$ & $14.4 \%$ & $100.0 \%$ \\
\hline & 2020-04 & $53.3 \%$ & $17.1 \%$ & $9.6 \%$ & $9.4 \%$ & $10.5 \%$ & $100.0 \%$ \\
\hline & $2020-05$ & $20.0 \%$ & $45.0 \%$ & $20.0 \%$ & $5.0 \%$ & $10.0 \%$ & $100.0 \%$ \\
\hline & $2020-06$ & $100.0 \%$ & & & & & $100.0 \%$ \\
\hline & $2020-08$ & & $25.0 \%$ & $27.1 \%$ & $47.2 \%$ & $0.7 \%$ & $100.0 \%$ \\
\hline & $2020-09$ & & $42.0 \%$ & $18.0 \%$ & $28.0 \%$ & $12.0 \%$ & $100.0 \%$ \\
\hline & $2020-10$ & $20.0 \%$ & $60.0 \%$ & & $20.0 \%$ & & $100.0 \%$ \\
\hline & $2020-11$ & $62.5 \%$ & & $25.0 \%$ & $12.5 \%$ & & $100.0 \%$ \\
\hline & 2021-01 & & $40.0 \%$ & $20.0 \%$ & $40.0 \%$ & & $100.0 \%$ \\
\hline & 2021-02 & & $6.3 \%$ & & $93.8 \%$ & & $100.0 \%$ \\
\hline & 2021-03 & $50.0 \%$ & & & & $50.0 \%$ & $100.0 \%$ \\
\hline & 2021-04 & $25.0 \%$ & & & $75.0 \%$ & & $100.0 \%$ \\
\hline & 2021-08 & $2.3 \%$ & $23.9 \%$ & $19.4 \%$ & $54.3 \%$ & & $100.0 \%$ \\
\hline & 2021-09 & $0.2 \%$ & $11.4 \%$ & $15.4 \%$ & $73.1 \%$ & & $100.0 \%$ \\
\hline & $2021-10$ & $0.5 \%$ & $37.0 \%$ & $19.4 \%$ & $36.7 \%$ & $6.4 \%$ & $100.0 \%$ \\
\hline Total & & $18.7 \%$ & $23.5 \%$ & $16.3 \%$ & $35.1 \%$ & $6.4 \%$ & $100.0 \%$ \\
\hline
\end{tabular}

In some periods there has been a substantial backlog of 'historical' cases.

\section{period * Historical Crosstabulation}

$\%$ within period

\begin{tabular}{lrr|r|r} 
& \multicolumn{3}{c|}{ Historical } & \\
\hline period & & \multicolumn{1}{c}{ Yes } & \multicolumn{1}{c}{ Total } \\
\hline \multirow{7}{*}{$2020-03$} & $100.0 \%$ & & $100.0 \%$ \\
\hline $2020-04$ & $100.0 \%$ & & $100.0 \%$ \\
\hline $2020-05$ & $95.0 \%$ & $5.0 \%$ & $100.0 \%$ \\
\hline $2020-06$ & $100.0 \%$ & & $100.0 \%$ \\
\hline $2020-07$ & $100.0 \%$ & & $100.0 \%$ \\
\hline
\end{tabular}




\begin{tabular}{|r|r|r|r}
\hline $2020-08$ & $100.0 \%$ & & $100.0 \%$ \\
\hline $2020-09$ & $98.9 \%$ & $1.1 \%$ & $100.0 \%$ \\
\hline $2020-10$ & $98.3 \%$ & $1.7 \%$ & $100.0 \%$ \\
\hline $2020-11$ & $95.1 \%$ & $4.9 \%$ & $100.0 \%$ \\
\hline $2020-12$ & $79.4 \%$ & $20.6 \%$ & $100.0 \%$ \\
\hline $2021-01$ & $82.7 \%$ & $17.3 \%$ & $100.0 \%$ \\
\hline $2021-03$ & $82.4 \%$ & $17.6 \%$ & $100.0 \%$ \\
\hline $2021-04$ & $95.0 \%$ & $5.0 \%$ & $100.0 \%$ \\
\hline $2021-05$ & $92.2 \%$ & $7.8 \%$ & $100.0 \%$ \\
\hline $2021-07$ & $70.4 \%$ & $29.6 \%$ & $100.0 \%$ \\
\hline $2021-08$ & $73.6 \%$ & $26.4 \%$ & $100.0 \%$ \\
\hline $2021-09$ & $78.6 \%$ & $21.4 \%$ & $100.0 \%$ \\
\hline $2021-10$ & $97.4 \%$ & $2.6 \%$ & $100.0 \%$ \\
\hline Total & $95.1 \%$ & $4.9 \%$ & $100.0 \%$ \\
\hline
\end{tabular}

\section{Community Cases - Period * Sex Crosstabulation}

$\%$ within period

\begin{tabular}{|c|c|c|c|c|c|}
\hline & \multicolumn{3}{|c|}{ Sex } & \multirow[b]{2}{*}{ Total } \\
\hline & & Female & Male & Unknown & \\
\hline \multirow[t]{15}{*}{ period } & 2020-03 & $54.6 \%$ & $45.4 \%$ & & $100.0 \%$ \\
\hline & 2020-04 & $56.5 \%$ & $43.5 \%$ & & $100.0 \%$ \\
\hline & 2020-05 & $65.0 \%$ & $35.0 \%$ & & $100.0 \%$ \\
\hline & 2020-06 & $100.0 \%$ & & & $100.0 \%$ \\
\hline & 2020-08 & $47.9 \%$ & $52.1 \%$ & & $100.0 \%$ \\
\hline & 2020-09 & $66.0 \%$ & $34.0 \%$ & & $100.0 \%$ \\
\hline & $2020-10$ & $20.0 \%$ & $80.0 \%$ & & $100.0 \%$ \\
\hline & $2020-11$ & $75.0 \%$ & $25.0 \%$ & & $100.0 \%$ \\
\hline & 2021-01 & $60.0 \%$ & $40.0 \%$ & & $100.0 \%$ \\
\hline & 2021-02 & $75.0 \%$ & $25.0 \%$ & & $100.0 \%$ \\
\hline & 2021-03 & $50.0 \%$ & $50.0 \%$ & & $100.0 \%$ \\
\hline & 2021-04 & $75.0 \%$ & $25.0 \%$ & & $100.0 \%$ \\
\hline & 2021-08 & $53.5 \%$ & $46.5 \%$ & & $100.0 \%$ \\
\hline & 2021-09 & $50.8 \%$ & $49.2 \%$ & & $100.0 \%$ \\
\hline & $2021-10$ & $48.1 \%$ & $51.7 \%$ & $0.2 \%$ & $100.0 \%$ \\
\hline Total & & $52.4 \%$ & $47.6 \%$ & $0.1 \%$ & $100.0 \%$ \\
\hline
\end{tabular}




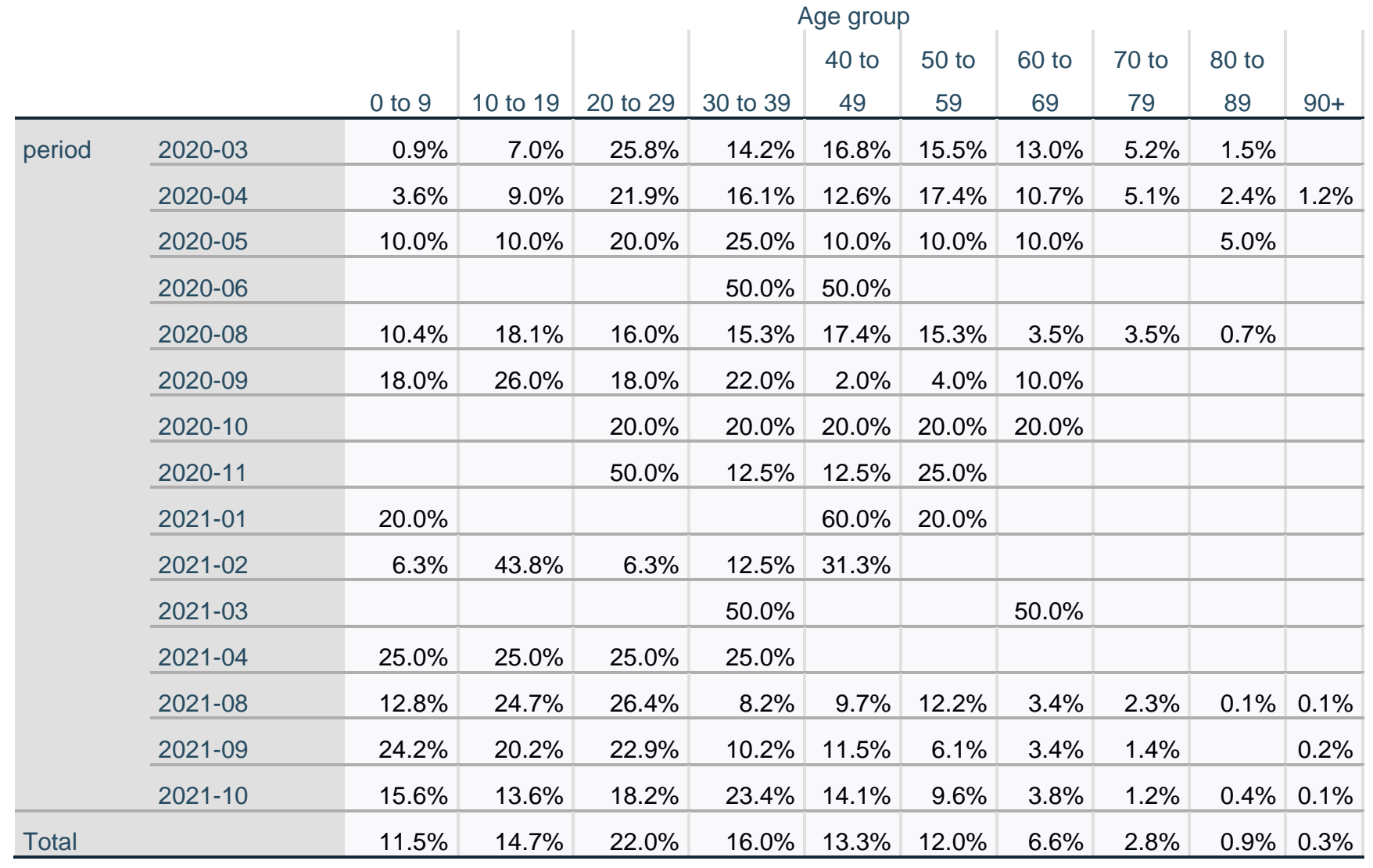

Period * Overseas travel Crosstabulation

\begin{tabular}{|c|c|c|c|c|c|}
\hline & & \multicolumn{3}{|c|}{ Overseas travel connection $\%$ within period } & \multirow[b]{2}{*}{ Total } \\
\hline & & No & Unknown & Yes & \\
\hline \multirow[t]{15}{*}{ period } & $2020-03$ & $45.3 \%$ & $0.4 \%$ & $54.3 \%$ & $100.0 \%$ \\
\hline & $2020-04$ & $77.1 \%$ & $0.3 \%$ & $22.7 \%$ & $100.0 \%$ \\
\hline & $2020-05$ & $85.0 \%$ & & $15.0 \%$ & $100.0 \%$ \\
\hline & $2020-06$ & & & $100.0 \%$ & $100.0 \%$ \\
\hline & $2020-08$ & $100.0 \%$ & & & $100.0 \%$ \\
\hline & $2020-09$ & $98.0 \%$ & $2.0 \%$ & & $100.0 \%$ \\
\hline & $2020-10$ & $100.0 \%$ & & & $100.0 \%$ \\
\hline & $2020-11$ & $100.0 \%$ & & & $100.0 \%$ \\
\hline & 2021-01 & $100.0 \%$ & & & $100.0 \%$ \\
\hline & 2021-02 & $100.0 \%$ & & & $100.0 \%$ \\
\hline & $2021-03$ & $50.0 \%$ & & $50.0 \%$ & $100.0 \%$ \\
\hline & 2021-04 & $100.0 \%$ & & & $100.0 \%$ \\
\hline & 2021-08 & $99.3 \%$ & $0.7 \%$ & & $100.0 \%$ \\
\hline & 2021-09 & $99.7 \%$ & $0.3 \%$ & & $100.0 \%$ \\
\hline & 2021-10 & $89.0 \%$ & $11.0 \%$ & & $100.0 \%$ \\
\hline Total & & $83.0 \%$ & $3.6 \%$ & $13.4 \%$ & $100.0 \%$ \\
\hline
\end{tabular}




\section{Period * Historical Crosstabulation}

$\%$ within period

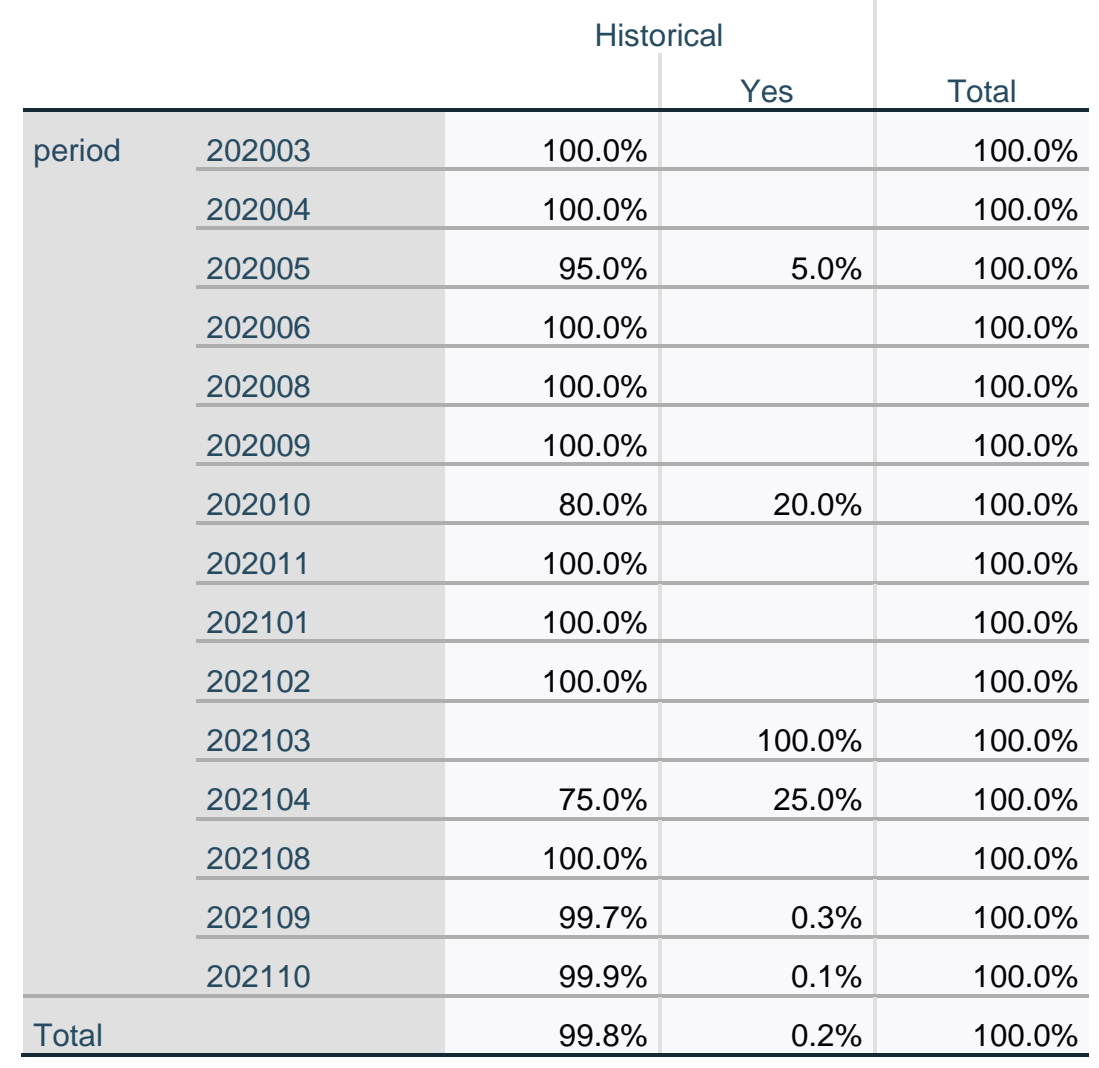

\section{Crosstabs MIQ Cases}

period * Sex Crosstabulation

$\%$ within period

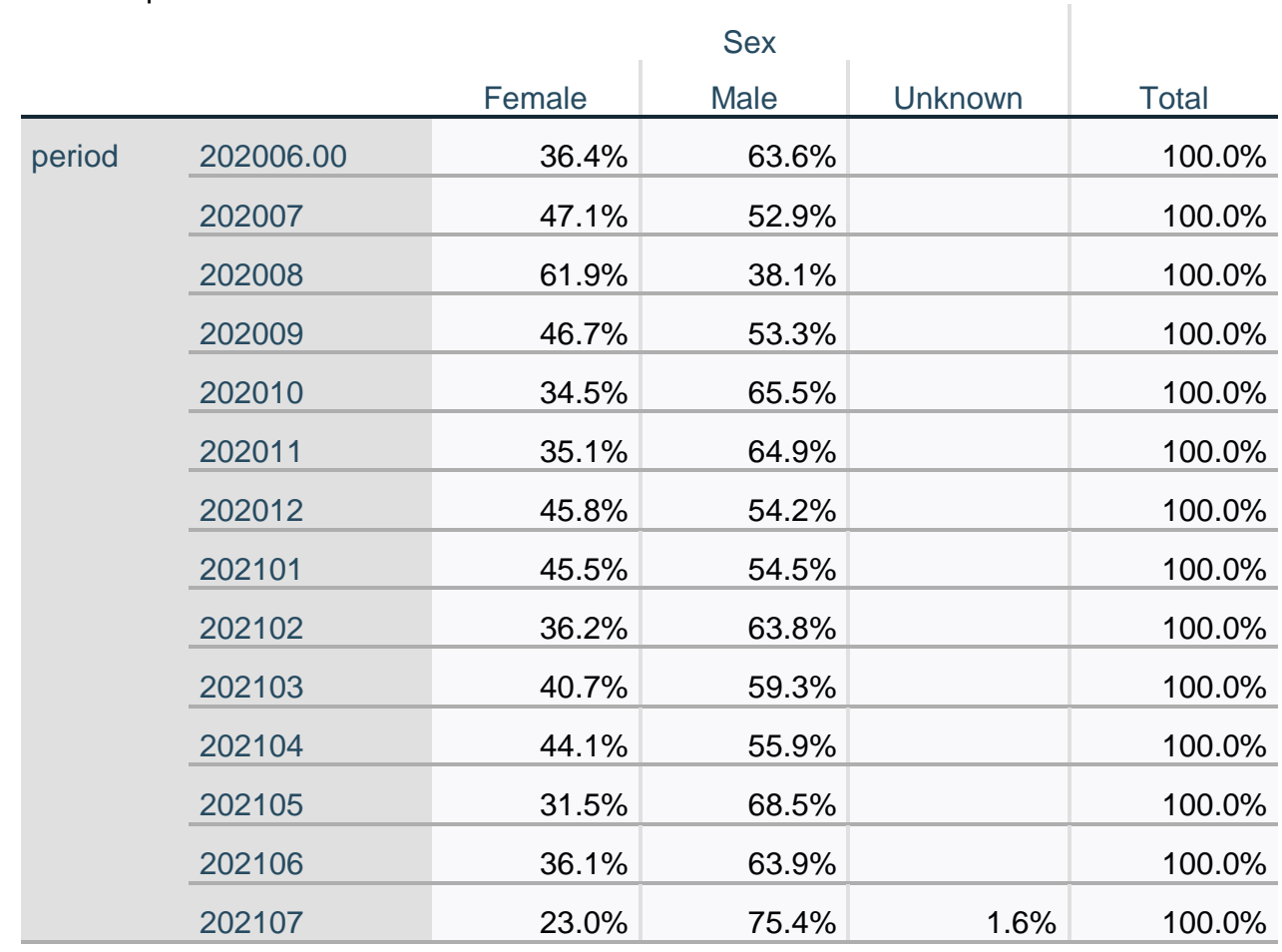




\begin{tabular}{|c|c|c|c|c|}
\hline 202108 & $36.4 \%$ & $63.6 \%$ & & $100.0 \%$ \\
\hline 202109 & $28.8 \%$ & $71.2 \%$ & & $100.0 \%$ \\
\hline 202110 & $34.2 \%$ & $65.8 \%$ & & $100.0 \%$ \\
\hline Total & $38.3 \%$ & $61.5 \%$ & $0.2 \%$ & $100.0 \%$ \\
\hline
\end{tabular}

\section{period * Age group Crosstabulation}

$\%$ within period

\begin{tabular}{|c|c|c|c|c|c|c|c|c|c|c|c|}
\hline & & & & & & group & & & & & \\
\hline & & 0 to 9 & $\begin{array}{c}10 \text { to } \\
19 \\
\end{array}$ & $\begin{array}{c}20 \text { to } \\
29 \\
\end{array}$ & $\begin{array}{c}30 \text { to } \\
39 \\
\end{array}$ & $\begin{array}{c}40 \text { to } \\
49 \\
\end{array}$ & 50 to 59 & $\begin{array}{c}60 \text { to } \\
69 \\
\end{array}$ & $\begin{array}{c}70 \text { to } \\
79 \\
\end{array}$ & $\begin{array}{c}80 \text { to } \\
89\end{array}$ & Total \\
\hline period & 202006 & $4.5 \%$ & $4.5 \%$ & $31.8 \%$ & $36.4 \%$ & & $9.1 \%$ & $9.1 \%$ & $4.5 \%$ & & $100.0 \%$ \\
\hline & 202007 & $2.9 \%$ & & $35.3 \%$ & $32.4 \%$ & $8.8 \%$ & $8.8 \%$ & $5.9 \%$ & $5.9 \%$ & & $100.0 \%$ \\
\hline & 202008 & $9.5 \%$ & $4.8 \%$ & $26.2 \%$ & $33.3 \%$ & $7.1 \%$ & $14.3 \%$ & $2.4 \%$ & & $2.4 \%$ & $100.0 \%$ \\
\hline & 202009 & $17.8 \%$ & $6.7 \%$ & $26.7 \%$ & $24.4 \%$ & $6.7 \%$ & $6.7 \%$ & $6.7 \%$ & $4.4 \%$ & & $100.0 \%$ \\
\hline & 202010 & $6.0 \%$ & $6.0 \%$ & $22.4 \%$ & $29.3 \%$ & $15.5 \%$ & $10.3 \%$ & $10.3 \%$ & & & $100.0 \%$ \\
\hline & 202011 & $3.2 \%$ & $12.8 \%$ & $29.8 \%$ & $21.3 \%$ & $12.8 \%$ & $9.6 \%$ & $7.4 \%$ & $3.2 \%$ & & $100.0 \%$ \\
\hline & 202012 & $4.7 \%$ & $9.3 \%$ & $21.5 \%$ & $28.0 \%$ & $18.7 \%$ & $10.3 \%$ & $5.6 \%$ & $1.9 \%$ & & $100.0 \%$ \\
\hline & 202101 & $9.0 \%$ & $9.0 \%$ & $26.1 \%$ & $28.4 \%$ & $14.2 \%$ & $8.2 \%$ & $4.5 \%$ & $0.7 \%$ & & $100.0 \%$ \\
\hline & 202102 & $5.2 \%$ & $8.6 \%$ & $20.7 \%$ & $25.9 \%$ & $19.0 \%$ & $17.2 \%$ & $3.4 \%$ & & & $100.0 \%$ \\
\hline & 202103 & $5.9 \%$ & $4.2 \%$ & $32.2 \%$ & $36.4 \%$ & $8.5 \%$ & $11.0 \%$ & $1.7 \%$ & & & $100.0 \%$ \\
\hline & 202104 & $11.7 \%$ & $2.7 \%$ & $29.7 \%$ & $28.8 \%$ & $11.7 \%$ & $7.2 \%$ & $2.7 \%$ & $3.6 \%$ & $1.8 \%$ & $100.0 \%$ \\
\hline & 202105 & $9.3 \%$ & $5.6 \%$ & $16.7 \%$ & $29.6 \%$ & $13.0 \%$ & $18.5 \%$ & $5.6 \%$ & $1.9 \%$ & & $100.0 \%$ \\
\hline & 202106 & $15.3 \%$ & $8.3 \%$ & $25.0 \%$ & $20.8 \%$ & $15.3 \%$ & $11.1 \%$ & $4.2 \%$ & & & $100.0 \%$ \\
\hline & 202107 & $8.7 \%$ & $2.4 \%$ & $20.6 \%$ & $27.8 \%$ & $20.6 \%$ & $14.3 \%$ & $4.0 \%$ & $1.6 \%$ & & $100.0 \%$ \\
\hline & 202108 & $11.4 \%$ & $13.6 \%$ & $22.7 \%$ & $20.5 \%$ & $15.9 \%$ & $8.0 \%$ & $6.8 \%$ & $1.1 \%$ & & $100.0 \%$ \\
\hline & 202109 & $13.6 \%$ & $6.1 \%$ & $34.8 \%$ & $21.2 \%$ & $6.1 \%$ & $16.7 \%$ & & $1.5 \%$ & & $100.0 \%$ \\
\hline & 202110 & $10.5 \%$ & $13.2 \%$ & $26.3 \%$ & $15.8 \%$ & $10.5 \%$ & $15.8 \%$ & $5.3 \%$ & $2.6 \%$ & & $100.0 \%$ \\
\hline Total & & $8.6 \%$ & $7.0 \%$ & $25.9 \%$ & $27.2 \%$ & $13.4 \%$ & $11.2 \%$ & $4.9 \%$ & $1.6 \%$ & $0.2 \%$ & $100.0 \%$ \\
\hline
\end{tabular}

\section{period * Overseas travel Crosstabulation}

$\%$ within period

\begin{tabular}{l|l|l|r|r} 
& \multicolumn{3}{c}{ Overseas travel } & \\
\hline period & No & Unknown & \multicolumn{1}{c}{ Yes } & \multicolumn{1}{c}{ Total } \\
\hline 202006 & & & $100.0 \%$ & $100.0 \%$ \\
\hline 202007 & & & $100.0 \%$ & $100.0 \%$ \\
\hline 202008 & & & $100.0 \%$ & $100.0 \%$ \\
\hline 202009 & $2.2 \%$ & $97.8 \%$ & $100.0 \%$ \\
\hline 202010 & $1.7 \%$ & $98.3 \%$ & $100.0 \%$ \\
\hline 202011 & $2.1 \%$ & $97.9 \%$ & $100.0 \%$ \\
\hline 202012 & & & $100.0 \%$ & $100.0 \%$ \\
\hline
\end{tabular}




\begin{tabular}{|r|r|r|r|r}
\hline 202101 & $0.7 \%$ & $99.3 \%$ & $100.0 \%$ \\
\hline 202102 & $1.7 \%$ & $98.3 \%$ & $100.0 \%$ \\
\hline 202103 & $2.5 \%$ & $97.5 \%$ & $100.0 \%$ \\
\hline 202104 & $3.6 \%$ & & $96.4 \%$ & $100.0 \%$ \\
\hline 202105 & & & $100.0 \%$ & $100.0 \%$ \\
\hline 202106 & $1.4 \%$ & & $98.6 \%$ & $100.0 \%$ \\
\hline 202107 & & & $100.0 \%$ & $100.0 \%$ \\
\hline 202108 & $1.1 \%$ & $98.9 \%$ & $100.0 \%$ \\
\hline 202109 & $3.0 \%$ & & $97.0 \%$ & $100.0 \%$ \\
\hline 202110 & & & $94.7 \%$ & $100.0 \%$ \\
\hline Total & $1.4 \%$ & $0.2 \%$ & $98.5 \%$ & $100.0 \%$ \\
\hline
\end{tabular}

7 MIQ:

There is limited information on MIQ.

Occupancy and returnee overview, as at 11:59PM 21 October 2021

Number of people currently in Managed Isolation facilities 4,867

Number of people currently in Quarantine facilities 343

Projected Returnees - Next 14 days 4,216

Total number of people through MIQ facilities since 26 March 2020 182,536

\section{International Comparisons}

Several agencies have ranked countries' Covid responses. NZ is depicted as having fallen from first place in their first ranking to $32^{\text {nd2 }}$ On the Bloomberg resilience rating largely as a result of the lockdown regime imposed in order to combat Delta. World-level comparative datasets and analyses can be found at:

- World Health Organization (WHO) COVID-19 dashboard

- John Hopkins Coronavirus Resource Center COVID-19 dashboard and map

- Worldometer COVID-19 statistics.

A useful presentation provides data for total cases, and then cases, deaths and tests per million population. It is important to exclude various cases to obtain more comparable measurement and to recognize that countries vary enormously in the accuracy of their reporting. Ranking (in my view) is best carried out on case rate. On this basis NZ is $3^{\text {rd }}$ amongst larger countries (after China and Taiwan and we do slightly better in terms of death rate.

\begin{tabular}{|c|c|c|c|c|c|}
\hline $\begin{array}{c}\text { Country, } \\
\text { Other }\end{array}$ & $\begin{array}{l}\text { Total } \\
\text { Cases }\end{array}$ & $\begin{array}{l}\text { Tot Cases/ } \\
\text { 1M pop }\end{array}$ & $\begin{array}{l}\text { Deaths/ } \\
1 \mathrm{M} \text { pop }\end{array}$ & $\begin{array}{l}\text { Tests/ } \\
\text { 1M pop }\end{array}$ & Population \\
\hline World & $237,548,427$ & 30,475 & 622.1 & & \\
\hline Micronesia & & & & & $\underline{116,548}$ \\
\hline Vanuatu & & & 3 & 72,738 & $\underline{316,202}$ \\
\hline$\underline{\text { Samoa }}$ & & 3 & & & $\underline{200,082}$ \\
\hline
\end{tabular}

\footnotetext{
${ }^{2}$ https://www.bloomberg.com/graphics/covid-resilience-ranking/
} 


\begin{tabular}{|c|c|c|c|c|c|}
\hline $\begin{array}{c}\text { Country, } \\
\text { Other }\end{array}$ & $\begin{array}{l}\text { Total } \\
\text { Cases }\end{array}$ & $\begin{array}{l}\text { Tot Cases/ } \\
1 \mathrm{M} \text { pop }\end{array}$ & $\begin{array}{l}\text { Deaths/ } \\
\text { 1M pop }\end{array}$ & $\begin{array}{l}\text { Tests/ } \\
\text { 1M pop }\end{array}$ & Population \\
\hline$\underline{\text { Solomon Islands }}$ & 20 & 28 & & 6,355 & $\underline{708,135}$ \\
\hline China & 96,357 & 67 & 3 & 111,163 & $1,439,323,776$ \\
\hline Palau & 5 & 275 & & 547,382 & $\underline{18,203}$ \\
\hline Taiwan & 16,271 & 682 & 35 & 271,139 & $\underline{23,871,339}$ \\
\hline New Zealand & 4,527 & 905 & 6 & 708,319 & $5,002,100$ \\
\hline Hong Kong & 12,252 & 1,618 & 28 & $3,378,368$ & $\underline{7,573,912}$ \\
\hline Papua New Guinea & 21,896 & 2,390 & 27 & 21,268 & $9,161,039$ \\
\hline Australia & 122,566 & 4,737 & 54 & $1,521,727$ & $\overline{25,873,925}$ \\
\hline$\overline{\text { Pakistan }}$ & $1,256,233$ & $\mathbf{5 , 5 5 1}$ & 124 & 87,414 & $2 \overline{26,308,104}$ \\
\hline Japan & $1,707,752$ & 13,555 & 141 & 199,945 & $\overline{125,986,140}$ \\
\hline Thailand & $1,689,437$ & 24,127 & 250 & 131,411 & $70,021,659$ \\
\hline India & $33,914,465$ & 24,274 & 322 & 414,167 & $\underline{1,397,161,207}$ \\
\hline$\underline{\text { New Caledonia }}$ & $\mathbf{8 , 8 6 0}$ & 30,661 & 640 & 147,961 & $\underline{288,969}$ \\
\hline Iceland & 12,092 & 35,147 & 96 & $3,133,372$ & $\underline{344,045}$ \\
\hline Norway & 192,587 & 35,177 & 159 & $1,450,078$ & $\underline{\mathbf{5 , 4 7 4 , 8 6 0}}$ \\
\hline$\overline{\text { Canada }}$ & $1,651,233$ & 43,271 & 737 & $1,154,798$ & $\overline{38,160,672}$ \\
\hline Germany & $4,306,757$ & 51,196 & 1,128 & 871,924 & $\underline{84,122,996}$ \\
\hline Russia & $7,690,110$ & 52,667 & 1,463 & $1,335,491$ & $146,013,681$ \\
\hline Fiji & 51,386 & 56,801 & 717 & 463,172 & $\underline{904,662}$ \\
\hline Denmark & 362,068 & 62,232 & 459 & $14,454,707$ & $\underline{5,818,016}$ \\
\hline$\underline{\operatorname{Iran}}$ & $5,674,083$ & 66,479 & 1,430 & 382,174 & $\underline{85,351,834}$ \\
\hline$\underline{\text { Italy }}$ & $4,692,274$ & 77,752 & 2,174 & $1,564,967$ & $\underline{60,349,253}$ \\
\hline Ireland & 397,831 & 79,447 & 1,054 & $1,516,543$ & $\overline{5,007,521}$ \\
\hline Austria & $\mathbf{7 5 5 , 7 9 7}$ & 83,316 & 1,221 & $9,868,576$ & $\underline{\mathbf{9 , 0 7 1 , 4 1 6}}$ \\
\hline Chile & $1,659,386$ & 85,873 & 1,942 & $1,148,861$ & $19,323,737$ \\
\hline Turkey & $7,357,336$ & 86,064 & 767 & 1,038,698 & $85,486,524$ \\
\hline Colombia & $4,967,524$ & 96,328 & 2,453 & 501,163 & $\underline{51,568,624}$ \\
\hline Switzerland & 847,451 & 97,021 & 1,274 & $1,268,104$ & $\underline{\mathbf{8 , 7 3 4 , 6 8 4}}$ \\
\hline Brazil & $21,532,558$ & 100,399 & 2,797 & 297,365 & $\underline{214,470,785}$ \\
\hline Portugal & $1,073,268$ & 105,646 & 1,774 & $1,857,349$ & $\underline{10,159,058}$ \\
\hline Spain & $4,971,310$ & 106,275 & 1,853 & $1,399,663$ & $\underline{46,777,704}$ \\
\hline France & $7,043,316$ & 107,603 & 1,787 & $2,188,250$ & $65,456,327$ \\
\hline Belgium & $1,256,191$ & 107,796 & 2,201 & $1,742,032$ & $11,653,450$ \\
\hline Sweden & $1,157,083$ & 113,674 & 1,460 & $1,251,982$ & $\underline{10,178,935}$ \\
\hline Argentina & $5,264,305$ & 115,142 & 2,524 & 530,461 & $\underline{45,720,260}$ \\
\hline Netherlands & $2,016,171$ & 117,336 & 1,059 & $1,018,230$ & $17,182,860$ \\
\hline$\underline{\mathbf{U K}}$ & $\mathbf{8 , 0 4 6 , 3 9 0}$ & 117,745 & 2,011 & $4,542,769$ & $\underline{68,337,222}$ \\
\hline$\underline{\text { USA }}$ & $45,021,267$ & 135,013 & 2,190 & $1,961,762$ & $\underline{\mathbf{3 3 3}, \mathbf{4 5 8 , 3 8 3}}$ \\
\hline French Polynesia & 40,178 & 141,991 & 2,212 & 93,140 & $\underline{282,962}$ \\
\hline$\underline{\text { Czechia }}$ & $1,697,064$ & 158,100 & 2,841 & $3,596,127$ & $\underline{10,734,129}$ \\
\hline
\end{tabular}




\section{Conclusions}

The data assembled in this research note shows that we are each experiencing Covid through the places we hold in society and viewpoints fatefully shaped by New Zealand's social order. But Covid is also fatefully reshaping this social order not just in the short term as we experience the current shifting phases but into the longer-term future.

These different ways we relate to Covid are fractured by underlying socio-economic, ethnic and age differences (and perhaps others). Managers and Professionals and Businesspeople (according to the Household Labourforce Survey data reported in Crothers, 2021) are more likely to be able to have the advantages of 'working from home' affording them more protection from being exposed to the virus - whereas many 'Essential workers' (see Appendix) that have needed to venture out are closer to the breadline (Stats NZ data). Those suffering from lower incomes are more likely to be causal workers. Many service workers are vulnerable, and many do not have the flexibility or even the transport to be able to easily take opportunities to be vaccinated. To the different opportunities and difficulties which accrue to different socio-economic situations must be added difficulties which can arise through ethnicity and language, some embedded in very long-standing experiences of deprivation and prejudice. Moreover many in difficult circumstances are further handicapped by their distance from facilities - and perhaps also the opportunities to talk with others about the situation and its dangers. Different ethnic groups also have different age-structures and the Ministry of Health data show that different age-cohorts are being vaccinated at fairly similar rates. Analysis has to dig below the surface.

The analysis of data from $\mathrm{MOH}$ show useful light on some of the underlying social differences at $\mathrm{DHB}$ and locality (SA2) levels. Vaccination rates are strongly shaped by social class, ethnicity and location. These points are important: although Māori lags in vaccination are partly due to the greater extent to which Māori are working class but there is also a major vaccination gap in working class areas in general - and also in those which are more remote.

A further new class division which is emerging is that between the vaccinated and the unvaccinated which hopefully at least will be temporary until more of the disadvantaged catch up in their vaccinations with the advantaged. Such a division seems unfortunately seems unavoidable to ensure we can all cope with Covid. Assigning extra resources to educate and assist the vaccine hesitant is essential and the Government is working on this. To keep tabs on this issue formal government reporting on progress is being carried out which is useful to agencies in targeting their efforts although unfortunately it adds further pressure and stigmatisation.

While the majority are law abiding and will follow rules designed to limit the dangers of Covid not all are, and appalled respectable citizens often cannot understand why simple rules (e.g. stay at home isolation) cannot be more widely followed, and perhaps don't realise how much extra resource is sucked into dealing with the relatively few stand-outs and why more complex approaches are needed. Covid too has brought out just how many people in various social situations (although many youth fall into this category) who are considerably detached from the broader society most respectable citizens live within.

Charting forward any path is completely rife with difficulty and continues to involve trade-offs in decisions and balancing of competing interests. The tendency of too many in the public, commentators in the media let alone opposition leaders is to paper over these conflicting interests and views and, worse, to increase polarizing them, which is not helpful to keeping the high social 
cohesion we need to get through these difficulties. I try here to document some of the ways in which New Zealanders are divided and deserve recognition and acceptance for difficulties

\section{References}

Crothers, Charles (2021) Research Note on Experiences and Attitudes Going Forward with and Beyond Delta (late-October Aotearoa New Zealand Journal of Social Issues. https://ojs.aut.ac.nz/anzjsi/article/

Jansen, Rawiri (2021) What New Zealand's huge gap in Covid outcomes tells us about systemic racism Spinoff https://thespinoff.co.nz/atea/09-07-2021/what-new-zealands-huge-gap-incovid-outcomes-tells-us-about-systemic-racism/

Saville-Smith, K and I. Mitchell (2020) Essential Workers' Bubbles: Crowding, Housing Affordability and Tenure https://www.buildingbetter.nz/publications/homes_spaces/SavilleSmith_Mitchell_May2020_crowding_affordability_stress_among_essential_workers.pdf

Steyn, Nicholas; Rachelle N Binny, Kate Hannah, Shaun C Hendy, Alex James, Audrey Lustig, Kannan Ridings, Michael J Plank, Andrew Sporle (2021) Māori and Pacific people in New Zealand have a higher risk of hospitalisation for COVID-19 New Zealand Medical Journal 9 July 2021, Vol 134 No 1538

ISSN 1175-8716

Steyn N, Binny RN, Hannah K, Hendy SC, James A, Kukutai T, et al. (2021) Estimated inequities in COVID-19 infection fatality rates by ethnicity for Aotearoa New Zealand. New Zealand Medical Journal. 2020;133(1520):28-39.

Whitehead, Jesse; Polly Atatoa Carr, Nina Scott, Ross Lawrenson (2021) Spatial inequity in distribution of COVID-19 vaccination services in Aotearoa medRxiv. doi: https://doi.org/10.1101/2021.08.26.21262647

Appendix: Essential Workers: Statistics NZ estimates by Occupation and Industry - underlying data from Saville-Smith \& Mitchell (2020).

\begin{tabular}{|l|l|l|}
\hline Occupation/Industry & Code & Numbers \\
\hline Checkout operators & 631111 & $16 \mathrm{k}$ \\
\hline Health carers & 423 & $68 \mathrm{k}$ \\
\hline Nurses and midwives & 254 & $58 \mathrm{k}$ \\
\hline Police officers & 4413 & $11 \mathrm{k}$ \\
\hline Rubbish \& recyclers & 8996 and 839918 & $1 \mathrm{k}$ \\
\hline Total Industry & & 156 \\
\hline 2. & & \\
\hline Supermarket \&rocery & $\mathrm{G} 411$ & $83 \mathrm{k}$ \\
\hline Health care care & $084 \&$ \& & $153 \mathrm{k}$ \\
\hline Residential & 286 & $7 \mathrm{k}$ \\
\hline Police & 07711 & $13.5 \mathrm{k}$ \\
\hline Waste management & $\mathrm{D} 291$ & $2 \mathrm{k}$ \\
\hline Total & & 258.5 \\
\hline
\end{tabular}

